\title{
Overexpression of sperm associated antigen 5 promotes cell growth, metastasis, and adriamycin resistance in esophageal squamous cell carcinoma via activating PI3K/AKT signaling pathway
}

\section{Yan Yan}

the first affiliated hospital of zhengzhou university

Fang bin Zhang

the first affiliated hospital of zhengzhou university

Qiao li Yi

the first affiliated hospital of zhengzhou university

Kun Zhou ( $\nabla$ zhoukunzkzz90@163.com )

the first affiliated hospital of zhengzhou university

Research

Keywords: SPAG5, growth, invasion, migration, adriamycin resistance, ESCC, PI3K/AKT

Posted Date: May 18th, 2020

DOI: https://doi.org/10.21203/rs.3.rs-28653/v1

License: (c) (1) This work is licensed under a Creative Commons Attribution 4.0 International License.

Read Full License 


\section{Abstract}

Background: Esophageal cancer (ESCC) is one of the most common malignant tumors in the digestive system. This study aims to explore the effects of sperm associated antigen 5 (SPAG5) on cell growth, metastasis, and azithromycin resistance in esophagus cancer and its molecular mechanism.

Methods: The DEGs were obtained from GSE92396, GSE17351, and GSE9982 datasets about ESCC. The PPI network was constructed using the STRING database and was visualized using Cytoscape software. The cytohubba plug-in of Cytoscape software was used to identify the hub genes of the PPI network. The DEGs were used to perform GO and KEGG pathway enrichment analysis using the DAVID database. Statistical analysis was performed to test the clinical and prognostic significance of SPAG5. Cell viability, proliferation, apoptosis, migration, and invasion were detected using CCK-8, colony formation, flow cytometry, transwell and scratch-wound assays. The expression of related genes was detected by qRTPCR, western blot and IHC assays. The oncogenicity of SPAG5 in ESCC cells was determined using the nude mouse transplantation tumor experiment.

Results: Ninety-three overlapping genes from the DEGs were used to construct the PPI network, and mainly enriched in BP, CC, and MF terms. COX regression analysis of OS showed that SPAG5 expression and $\mathrm{pN}$ category were correlated with OS. Univariate and multivariate analyses showed that SPAG5 was an independent prognostic factor for OS in ESCC. The ROC curve analysis showed the AUC of SPAG5 was 0.74. Multiple logistic regression showed that SPAG5 were subsequently identified as an independent risk factor associated with OS. SPAG5 overexpression was detected in ESCC tissues and cell lines, and improved cell proliferation. SPAG5 knockdown reduced cell growth and metastasis and promoted its apoptosis. The functions of SPAG5 overexpression promoting ESCC cell growth and affecting cleaved caspase-3, Ki67, VEGF, and MMP-2/-9 expression were reversed by PI3K/AKT inhibitor. SPAG5 overexpression enhanced resistance to ADM in EC9706 and Eca109 cells and it was closely related to the activation of PI3K/AKT signaling pathway.

Conclusion: The overexpression of SPAG5 was an independent good prognostic factor and promoted the proliferation, invasion, migration, and ADM resistance, and inhibited the apoptosis via activating $\mathrm{PI3K} / \mathrm{AKT}$ signaling pathway in ESCC.

\section{Introduction}

Esophageal cancer (ESCC) is a kind of malignant tumor of digestive system which occurs in esophageal epithelial tissue [1]. The histopathological types of esophageal cancer can be divided into two types, one is ESCC [2], the other is esophageal adenocarcinoma [3]. China has a high incidence of ESCC [4]. Epidemiological studies have shown that smoking, drinking, lack of nutrients and obesity are closely related to the incidence of esophageal cancer. If early esophageal cancer is diagnosed in time and radical operation is performed, the prognosis is good, the 5-year postoperative survival rate can reach more than $90 \%$ [5]. Most patients with ESCC have developed into late stage after clinical symptoms and the 
prognosis is very poor, and the 5-year postoperative survival rate is less than $20 \%$ [6]. Esophageal cancer usually has no obvious symptoms in the early stage, such as dysphagia or weight loss without obvious reasons, the preliminary diagnosis is usually made by endoscopy. After the cancer was diagnosed, endoscopic ultrasonography was used to determine the depth of the tumor and evaluate whether lymph nodes were involved [7]. computed tomography (CT) was used to rule out distant metastasis and determine the initial stage. Carcinoma in situ can be treated by endoscopic mucosal resection, while local tumors with or without regional lymph node metastasis can be treated by esophagectomy, chemotherapy, chemotherapy or combination therapy $[8,9]$. Unresectable tumors or tumors with distant metastases outside the region are treated with palliative interventional therapy [10]. At present, there is no definite prevention strategy and early screening for esophageal cancer. The transcriptional regulation of cellular genes is very important for the normal development of cells. The changes of normal transcriptional factors can lead to abnormal proliferation or apoptosis and can lead to pathological changes of malignant proliferation of biological cells. Understanding the normal process of transcriptional regulation of transcriptional factors and the changes of transcriptional factor levels under pathological conditions can provide more evidence for targeted therapy of tumors.

The abnormal cell proliferation is an important mechanism of tumorigenesis [11]. In the process of cell mitosis, the abnormal structure and function of spindle and centromere are important factors for the abnormal cell proliferation. Sperm associated antigen5 (SPAG5) gene is located on chromosome Ch17q11.2, is one of the spindle binding proteins, which regulates spindle assembly time and sister chromosome separation during mitosis [12]. In S phase of cell division, SPAG5 can enter the spindle by binding to the C-terminal of DNA and regulate the spindle until the end of cell division [13]. SPAG5 mainly affects cell proliferation through microtubule structure, and its expression affects the efficacy of chemotherapeutic drugs. Many literatures have shown that SPAG5 is abnormally expressed in human tumors and plays an important role in tumorigenesis and development $[14,15,16,17]$. For example, SPAG5 is significantly upregulated in pancreatic cancer [18], renal, liver cancer [14], cervical cancer [16], non-small cell lung cancer [15] and prostate cancer [19]. Overexpression of SPAG5 promotes the proliferation of tumor cells and inhibits their apoptosis, which is closely related to the poor prognosis of tumor patients. According to the results of bioinformatics analysis and literature reports in our group, SPAG5 is an important hub gene and therapeutic target in esophageal cancer. However, the role and molecular mechanism of SPAG5 in esophageal cancer are not clear. Therefore, our team intends to explore the effect and molecular mechanism of SPAG5 on ESCC cell proliferation, apoptosis, migration, invasion and chemotherapy resistance from clinical, in vitro and animal models, so as to provide a new target for early diagnosis and treatment of esophageal cancer.

\section{Materials And Methods}

\section{Acquisition of gene expression profile microarray data}

The microarray data were acquired from the Gene Expression Omnibus (GEO) database (www.ncbi.nlm.nih.gov/geo). Three gene expression datasets (GSE9982, GSE17351, and GSE92396) of 
ESCC were included in this study.

GSE9982 dataset included 2 normal cells samples and 20 ESCC cells samples; GSE17351 dataset included 5 paired tumor and normal adjacent tissue samples; GSE92396 dataset included 9 normal adjacent tissue samples and 12 tumor tissue sample.

GSE9982 dataset was based on the platform of GPL1928 [CodeLink Human 20K ver4.1]; GSE17351 dataset was based on the platform of GPL570 [HG-U133_Plus_2] Affymetrix Human Genome U133 Plus 2.0 Array; GSE92396 dataset was based on the platform of GPL6244 [HuGene-1_0-st] Affymetrix Human Gene 1.0 ST Array [transcript (gene) version].

\section{Identification of DEGs}

The interactive web tool GEO2R (www.ncbi.nlm.nih.gov/geo/geo2r) was used to screen the DEGs between control group samples and ESCC group samples. The Benjamin and Hochberg false discovery rate (FDR) method was used to correct the adjusted $p$-value and correct the occurrence of false positive results. The cutoff standard was defined as $p$-value $<0.05$, adjusted $p$-value $<0.01$ and $|\log F C|>1$.

\section{GO terms and KEGG pathway enrichment analysis}

To identify the GO annotation and pathways of DEGs were enriched, GO terms and KEGG pathway enrichment analysis were perform using the Database for Annotation, Visualization and Integrated Discovery (DAVID) (https://david.ncifcrf.gov/). The significance level of KEGG pathway enrichment was calculated using a cutoff of $p$-value $<0.05$. GO term, including biological process (BP), cell component (CC) and molecular function (MF), was considered significantly enriched if it showed a $p$-value $<0.05$.

\section{Construction of the PPI network and identification of Hub gene}

The PPI network of DEGs was identified using the Search Tool for the Retrieval of Interacting Genes (STRING) (http://string-db.org/) database. Next, Cytoscape software (v 3.6.2) was used to visualize the PPI network. The cytohubba plug-in of cytoscape software was used to identify the top 10 hub genes of PPI network.

\section{Patients and tissues specimens}

A total of 42 cases of paired ESCC tissues and the corresponding adjacent nontumor tissues were collected immediately after surgery resection at The First Affiliated Hospital of Zhengzhou University from March 2016 to October 2018. Briefly, inclusion criteria were as follows: histologic proof of thoracic ESCC, complete surgical resection (R0), and complete follow-up data. The exclusion criteria included: received neoadjuvant or adjuvant treatment, history of other malignancy, death in perioperative period, and cervical lymph nodes metastases. The preoperative workup was evaluated by endoscopy with biopsy and histologic examination, barium esophagography to confirm tumor location, thoracic and abdominal computed tomography (CT), and cervical ultrasonography (with biopsy if indicated) if cervical lymph 
node metastasis was suspected. Histological differentiation, pT category (depth of tumor invasion), and $\mathrm{pN}$ category (lymph node metastasis) were determined by pathologic examination. Tissue samples were quickly frozen in liquid nitrogen after surgery and stored in $-80^{\circ} \mathrm{C}$ freezer. All patients gave written informed consent before operation. The study was approved by the Ethics Committee of the Cancer Center of The First Affiliated Hospital of Zhengzhou University. The clinical characteristics of the 67 ESCC patients are listed in Table 1 and Table 2.

\section{Cell culture}

The ESCC cell lines, including CaEs-17, TE-1, Eca109, KYSE520, KYSE30, EC9706, and TE-10 were obtained from ATCC. The normal esophageal epithelial cell lines, including HEEC and Het-1A were obtained from Suran biotechnology Co., Ltd (Shanghai, China). All cells were maintained in Dulbecco's Modified Eagle Medium supplemented with $10 \%$ fetal bovine serum and $10 \%$ penicillin-streptomycin at $37^{\circ} \mathrm{C}$ in a humidified incubator containing $5 \% \mathrm{CO}_{2}$.

\section{Establishment of ADM-resistant cells}

ADM-resistant cells Eca109/ADM and EC9706/ADM were incubated from parental Eca109 and EC9706 cells under continuous exposure to ADM with gradually increasing concentrations of docetaxel from 5 $\mathrm{nM}$ to $200 \mathrm{nM}$ in the culture medium. Briefly, Eca109 and EC9706 cells were incubated first with $5 \mathrm{nM}$ ADM for 2 days followed by incubation in the absence of ADM until the ADM-sensitive clones died. Then, survivable cells were cultured in the presence of $10 \mathrm{nM}$ Dox. The same procedure was repeated until cells that were viable at $200 \mathrm{nM}$ ADM were obtained. The surviving cells repopulated and after 10 months, the cells dividing freely in $10 \mathrm{nM}$ and $200 \mathrm{nM}$ ADM-containing media were considered as resistant cell lines and labeled as "Eca109/ADM" and "EC9706/ADM," respectively.

\section{Small interfering RNA (siRNA) transfection}

The siRNA against SPAG5 was prepared as described in a previous study [20]. A scrambled sequence unrelated to siSPAG5 was used as control. For transfection, Eca109, TE-10 and KYSE30 cells were plated in six-well plates containing $2 \mathrm{~mL}$ of the medium supplemented with $10 \%$ fetal bovine serum (FBS). After $18 \mathrm{~h}$, siSPAG5 was transfected using Lipofectamine ${ }^{\circledR} 2000$ Reagent (Invitrogen, Carlsbad, CA, USA) when the cells were $50 \%-60 \%$ confluent. The cells were harvested at $48 \mathrm{~h}$ after transfection and used for analysis.

\section{Plasmid Transfection}

SPAG5 and an empty vector (pcDNA 3.1) were purchased from Era Biotech (Shanghai, China). Lipofectamine 2000 (Invitrogen) was used to transfect 35 nM SPAG5 expression vector, or $35 \mathrm{nM}$ empty pcDNA3 vector (NC) into $2 \times 10^{5}$ cells. All subsequent experiments were carried out at $24 \mathrm{~h}$ after transfections.

\section{Colony formation assay}


Transfected cells were seeded in 6-well plates at 500 cells per well and cultured for 14 days. Cells were fixed with $4 \%$ paraformaldehyde solution (Beyotime Biotechnology, Shanghai, China), stained with $0.1 \%$ crystal violet (Beyotime), and counted under an inverted microscope.

\section{Cell viability assay}

The effects of SPAG5 on the viability of ESCC cells were measured by the CCK-8 assay (Beyotime). Briefly, cells were seeded in 96-well plates and cultured overnight. Cells were transfected with siRNA or plasmids for $6 \mathrm{~h}$, and the medium was replaced. The absorbance at $450 \mathrm{~nm}$ was measured using a microplate reader at $0,12,24,48$, and $72 \mathrm{~h}$.

\section{Cell apoptosis assay}

The cells were trypsinized after $24 \mathrm{~h}, 48 \mathrm{~h}$ and $72 \mathrm{~h}$ of incubation, $1 \times 10^{6}$ cells per sample were counted and washed twice by ice-cold PBS solution, and then $5 \mu \mathrm{L}$ of Annexin V-FITC and $10 \mu \mathrm{L}$ of propidium iodide (PI) (Sigma, MO, USA) were added to $100 \mu \mathrm{L}$ of cell suspension, followed by incubation for 15 min at room temperature in the dark. Finally, $400 \mu \mathrm{L}$ of binding buffer was added to each sample and filtered by 300-mesh nylon net before EPICS XL (Beckman Coulter, Bren CA USA) flow cytometer. EXPO32 ADC Analysis Software was used to analyze the data.

\section{Western blot assay}

Total protein of cells transfected with siRNA or plasmids was extracted by cell lysis in RIPA buffer (Thermo Fisher Scientific, MA, USA) containing protease and phosphatase inhibitor cocktails (Bimake, Shanghai, China). The protein concentrations were detected by a BCA Protein Assay Kit (Invitrogen). Proteins were separated by SDS-PAGE, transferred onto polyvinylidene difluoride (PVDF) membranes (Millipore, Darmstadt, Germany). After blocking by $5 \%$ non-fat milk, the membrane was incubated with different primary antibodies including SPAG5 (1:500; sc-100885; Santa Cruz Biotechnology; USA), cleaved-caspase-3 (1:800; ab2302; Abcam; Cambridge; UK), Ki67 (1:1000; ab16667; Abcam), VEGF (1:1000; ab32152; Abcam); MMP-2 (1:1200; ab92536; Abcam), MMP-9 (1:800; ab119906; Abcam), PI3K (1:1000; AF7742; Beyotime), AKT (1:800; AF1777; Abcam), phosph-PI3K (1:500; AF5905; Beyotime), phosph-AKT (1:500; AF1546; Abcam) and GAPDH (1:2000; AF5009; Abcam). The signals were detected by an HRP-conjugated secondary antibody (1:2000 dilution. Cell Signaling Technology) and the bands were visualized with an enhanced chemiluminescence (ECL, Millipore) system according to the manufacturer's protocol.

\section{RNA Extraction and qRT-PCR}

Total RNA was extracted with TRIzol reagent (Invitrogen, Carlsbad, CA, USA) according to the manufacturer's instructions. cDNA was amplified by qRT-PCR with SYBR Green (TaKaRa, Tokyo, Japan). The expression of target genes was normalized to the expression of the housekeeping gene 
glyceraldehyde-3-phosphate dehydrogenase (GAPDH). The following primers were used to detect the expression of SPAG5, CDC6, RAD51AP1, ETC2, and GAPDH:

SPAG5: 5囚- CGGGCGAATCCAAGATGATTC -3囚

5囚- AATGCCACATGTGCACATAGGA -3邓;

CDC6: 5囚-GCTTACGTGTCCGACTGCCCAAAAT -3囚

5囚- ATCCTCTCTCGAGAGAGTTAACTGGTT -3;

RAD51AP1: 5囚-TCCGAGAAACACACAAAGGGTCC -3囚

5囚- CTGGTGTCAACTGTCCATTTACCCAT-3;

ETC2: 5囚-TGGCTGTGCCTGTAAACACACCCCAA -3®

5囚- ACTGTGCAACTGCCATTTAAACGGGATT -3;

GAPDH: 5囚-GATCTTCGTTCGCGCCTTCGTC-3囚

5囚-GACGCGTTCAGTCCTCTTTCCG-3囚

\section{Scratch assay}

Cells were seeded into 6-well plate with the concentration of $3 \times 10^{5} / \mathrm{mL}$. After overnight culturing, wounds were created by using a $200 \mu \mathrm{L}$ pipet tip. Then the medium was changed to remove the detached cells. After culturing for $48 \mathrm{~h}$, image was captured by an inverted microscopy (Nikon, Tokyo, Japan) and the wound healing ability of each cell line was analyzed.

\section{Transwell assay}

The transwell chambers (Corning, NY, USA) with Matrigel were used to detect cell invasion and chambers without Matrigel were used to examine cell migration. $5 \times 10^{4}$ cells were seeded into the upper chambers of transwell in $200 \mu \mathrm{L}$ serum-free medium. $500 \mu \mathrm{L}$ medium containing 10\% FCS was used as the chemoattractant and added to the lower wells. After $24 \mathrm{~h}$, the chambers were fixed with $80 \%$ ethanol and stained with crystal violet $(20 \mathrm{mg} / \mathrm{mL})$. Then the cell numbers were counted in five individual random fields (200x) under a light microscope (Olympus, Tokyo, Japan), and the average cell density per field was calculated.

\section{ESCC cancer mouse xenograft model}

All the procedures of animal experiments were approved by the Animal Care and Use Committee of Zhengzhou University. 12 immunodeficient mice (BALB/C) were randomly separated into three groups: negative control (NC) group, SPAG5 overexpression group (SPAG5), and SPAG5+LY294002 group. After 
anesthesia, $5 \times 10^{6}$ cells transfected with NC or SPAG 5 overexpression plasmids were subcutaneously implanted into each immunodeficient mice. After $5 \times 10^{6}$ cells transfected with SPAG5 overexpression plasmids were subcutaneously implanted into each immunodeficient mice, PI3K/AKT inhibitor (LY294002; 1.5mg/kg/day; MedChemExpress) subcutaneously injected to each immunodeficient mice. Tumor samples were collected at 28 days after implantation.

\section{Immunohistochemistry assay}

Paraffin-embedded sections were baked at $70{ }^{\circ} \mathrm{C}$ overnight, then deparaffinized and rehydrated with xylene and ethanol. The sections were soaked in citrate buffer in a microwave for $15 \mathrm{~min}$. After recovering room temperature, the sections were treated with $0.3 \%$ hydrogen peroxide for 20 min to quench the endogenous peroxidase activity, and then incubated with a rabbit monoclonal anti-SPAG 5 antibody (1:500) overnight at $4{ }^{\circ} \mathrm{C}$. After washing with phosphate buffered saline (PBS), sections were incubated with secondary antibody. After washing with PBS, added a secondary antibody of undiluted horseradish peroxidase (HRP)-conjugated goat anti-rabbit to the sections at $37^{\circ} \mathrm{C}$ for $30 \mathrm{~min}$. The pathological sections were immersed in 3, 3'-Diaminobenzidine (DAB) for 3 minutes, counterstained with 10\% Mayer's hematoxylin, dehydrated. Two pathologists, unknown to the clinical result, scored the results independently. According to the staining intensities from light to general and dark brown, the expression levels were classified in three degrees: weakly, moderately, and strongly positive respectively.

\section{Statistical analysis}

SPSS 22.0 (SPSS Inc., Chicago, IL, USA) was used for statistical analysis. Quantitative variables were compared using the Student's $t$-test and expressed as median \pm standard deviation. The association between CDC6 expression, RAD51AP1 expression, ECT2 expression or SPAG5 expression and clinicopathological features of ESCC was analyzed by $\chi^{2}$ test. Survival analysis was performed using the Kaplan-Meier method, and differences in survival were estimated via log-rank test. Significant parameters revealed through univariate analysis were subjected to multivariate survival analysis by using Cox's proportional hazard model. The hazard ratios (HRs) and corresponding 95\% confidence intervals (Cls) were calculated. The ROC curve was used to evaluate the prognostic performance through comparing the areas under the ROC curves (AUC). The prognostic value of the CDC6 expression, RAD51AP1 expression, ECT2 expression, and SPAG5 expression was evaluated using multiple logistic regression with forward stepwise selection of variables. Two-tailed $p$-value $<0.05$ was considered statistically significant.

CDC6 mRNA expression <3.64 (average value) was described as low expression group while $\geq 3.64$ as high expression group; RAD51AP1 mRNA expression <4.82 (average value) was described as low expression group while $\geq 4.82$ as high expression group; ECT2 mRNA expression $<2.57$ (average value) was described as low expression group while $\geq 2.572$ as high expression group; SPAG 5 mRNA expression $<1.87$ (average value) was described as low expression group while $\geq 1.87$ as high expression group. 


\section{Results}

\section{Identification and analysis of DEGs in ESCC}

The ESCC RNA-seq data in the present study included GSE9982, GSE17351, and GSE92396 datasets. A total of 2242 DEGs from GSE9982 dataset were identified using the cut-off criteria of $p<0.05$ and |logFC| $>1$ (Figure 1a); A total of 2453 DEGs from GSE17351 dataset were identified using the cut-off criteria of $p$ $<0.05$ and $|\log \mathrm{FC}|>1$ (Figure 1b); A total of 1600 DEGs from GSE92396 dataset were identified using the cut-off criteria of $p<0.05$ and $|\log F C|>1$ (Figure 1c). There were 774 downregulated and 1468 upregulated in the DEGs of GSE9982 dataset (Figure 1d); There were 1152 downregulated and 1301 upregulated in the DEGs of GSE17351 dataset (Figure 1e); There were 790 downregulated and 810 upregulated in the DEGs of GSE92396 dataset (Figure 1f). A total of 93 overlapping genes from the DEGs of GSE9982, GSE17351, and GSE92396 datasets were identified using Draw Venn Diagram website (http://bioinformatics.psb.ugent.be/webtools/Venn/) (Figure 1g). GO term analysis showed 93 overlapping genes were mainly enriched in BP term (Figure 1h-1k), CC term (Figure 1l), and MF term (Figure 1m) using DAVID database (Figure 1n-10). KEGG pathway enrichment analysis showed that 93 overlapping genes were mainly related to "ECM-receptor interaction" and "Glycerolipid metabolism" (Figure 1p). The top 10 hub genes, including AURKA, PLK1, TPX2, CDC6, FOXM1, TRIP13, RAD51AP1, ECT2, SPAG5, and CKS1B, were obtained from the PPI network of 93 overlapping genes using STRING website and Cytoscape software (Figure 1q and 1r). In addition, the top 10 hub genes were significantly upregulated in ESCC based on the analysis of GSE9982, GSE17351, and GSE92396 datasets.

\section{SPAG5 expression was related to the clinicopathological characteristics of patients with ESCC}

To investigate the relationship between CDC6 expression, RAD51AP1 expression, ETC2 expression or SPAG5 expression and the clinicopathological features of ESCC patients. CDC6 expression, RAD51AP1 expression, and ETC2 expression was significantly associated with pT category and pN category, respectively ( $p<0.05$; Table 1 and Table 2 ) and was not associated with age, gender, location, differentiation, and pathological staging. SPAG5 expression was not associated with age, gender, location, differentiation, $\mathrm{pT}$ category, $\mathrm{pN}$ category and pathological staging ( $\mathrm{p}>0.05$; Table 1 and Table 2). The relationship between CDC6 expression level, RAD51AP1 expression level, ETC2 expression level, or SPAG5 expression level and patient survival was analyzed using Kaplan-Meier analysis (Figure 2a) and the log-rank test. Low levels of CDC6 expression, RAD51AP1 expression, ETC2 expression, or SPAG5 expression were significantly associated with a poorer OS than high expression levels of CDC6 expression, RAD51AP1 expression, ETC2 expression, or SPAG5 expression $(p<0.05)$. These results suggest that CDC6, RAD51AP1, ETC2 or SPAG5 is related to ESCC tumorigenesis. The univariate and multivariate analysis of COX regression for overall survival (Table 3) suggested that SPAG5 expression was an independent prognostic factor for ESCC ( $p=0.04$; Figure $2 b)$.

High SPAG5 expression was correlated with a high risk of OS in patients with ESCC 
Through logistic regression analysis, the association between MT1M expression and LNM was further evaluated. Eleven features were reduced to six potential predicators on the basis of 67 patients in the cohort ( 5:1 ratio; Figure $2 \mathrm{c}$ and $2 \mathrm{~d}$ ) and were with nonzero coefficients in the LASSO regression model. These features included location, pT category, pN category, pathological staging, ECT2 expression, and SPAG5 expression (Table 4). Logistic analysis showed that pT category, pN category, and high expression of SPAG5 are independent high-risk factors for OS in patients with ESCC (Table 4). The model that incorporated the above independent predictors was developed and presented as the nomogram (Figure 2e). ROC curve analysis showed that OS risk in patients with ESCC was $74 \%$ (Figure $2 \mathrm{f}$ ). It showed that SPAG5 expression has good predication ability for OS risk in patients with ESCC. The calibration curve of the OS risk nomogram for the prediction of OS risk in patients with ESCC demonstrated good agreement in this cohort (Figure 2g).

\section{High SPAG5 expression was detected in ESCC tumor tissues and cell lines}

Our results showed SPAG5 was significantly upregulated in tumor tissues of the patients with ESCC using qRT-PCR, IHC and western blot assays (Figure 3a-3c). Likewise, high SPAG5 expression was detected in ESCC cell lines compared to control group (HEEC and Het-1A cells) (Figure 3d and 3e). In addition, SPAG5 expression in CaEs-17, KYSE150, and EC9706 cells was significantly lower than that in other ESCC cell lines and higher than that in HEEC cells, and SPAG5 expression in Eca109 and TE-10 cells was significantly higher than in other ESCC cell lines and HEEC cells. SPAG5 expression in KYSE150 and EC9706 cells was significantly lower than that in other ESCC cell lines and higher than that in Het-1A cells, and SPAG5 expression in Eca109 and KYSE30 cells was significantly higher than in other ESCC cell lines and Het-1A cells. Therefore, Eca109, KYSE30, and TE-10 cells were used to perform small RNA interference experiment and KYSE150 and EC9706 cells were used to perform gene overexpression experiment.

\section{SPAG5 knockout inhibited the viability, proliferation, invasion and migration, and promoted the apoptosis, regulated proteins expression and suppressed the activation of PI3K/AKT signaling pathway in Eca109, KYSE30, and TE-10 cells}

After siRNA targeting SPAG5 was transfected into Eca109, KYSE30, and TE-10 cells, low SPAG5 expression was detected in siSPAG5 group using qRT-PCR and western blot assays (Figure 4a). The results of CCK-8 and clone formation assays confirmed that SPAG5 knockout inhibited the viability and proliferation of Eca109, KYSE30, and TE-10 cells (Figure 4b and 4c), whereas, SPAG5 knockout promoted their apoptosis using flow cytometry analysis (Figure 4d). Transwell and scratch-wound assays showed that SPAG5 knockout inhibited the invasion and migration of ESCC cells (Figure 4e-4f). Our results showed that cleaved caspase-3 expression in ESCC cells was increased by SPAG5 knockout and Ki67, VEGF, and MMP-2/-9 expression in ESCC cells were decreased by SPAG5 knockout (Figure 4g). Meanwhile, SPAG5 knockout significantly reduced the phosphorylation levels of PI3K and AKT (Figure 4h). 
SPAG5 overexpression promoted the viability, proliferation, invasion and migration, and suppressed the apoptosis, regulated proteins expression and activated PI3K/AKT signaling pathway in KYSE150 and EC9706 cells

After SPAG5 expressing plasmids were transfected into KYSE150 and EC9706 cells, high SPAG5 expression was detected in SPAG5 group using qRT-PCR and western blot assays (Figure 5a). The results of CCK-8 and clone formation assays confirmed that SPAG5 overexpression increased the viability and proliferation of KYSE150 and EC9706 cells (Figure 5b and 5c), whereas, SPAG5 overexpression inhibited their apoptosis using flow cytometry analysis (Figure 5d). Transwell and scratch-wound assays showed that SPAG5 overexpression promoted the invasion and migration of ESCC cells (Figure 5e-5f). Our results showed that cleaved caspase-3 expression in ESCC cells was reduced by SPAG5 overexpression and Ki67, VEGF, and MMP-2/-9 expression in ESCC cells were increased by SPAG5 overexpression (Figure 5g). Meanwhile, SPAG5 overexpression significantly upregulated the phosphorylation levels of PI3K and AKT (Figure 5h).

High SPAG5 expression promoted the growth and metastasis of ESCC in vivo and in vitro via activating PI3K/AKT signaling pathway

Our results showed that the functions of SPAG5 overexpression promoting the proliferation, viability, invasion, and migration and inhibiting its apoptosis in KYSE150 cells were reversed by PI3K/AKT inhibitor (LY294002) using CCK-8, clone formation, transwell, and scratch-wound assays and flow cytometry analysis (Figure 6a-6e). Next, LY294002 inhibited SPAG5-medicated cleaved caspase-3 protein expression decreased and Ki67, VEGF, and MMP-2/-9 protein expression increased in KYSE150 cells (Figure 6f). The nude mouse transplantation tumor experiment displayed that the oncogenicity of SPAG5 overexpression transfected KYSE150 cells was abolished by LY294002 (Figure 6g). IHC assay showed that the functions of SPAG 5 overexpression inhibiting cleaved caspase-3 expression and promoting MMP-2 and VEGF expression were reversed by LY294002 (Figure 6h).

\section{High SPAG5 expression was detected in ADM resistance of ESCC cells}

CCK-8 assay showed that the viability of EC9706/ADM and Eca109/ADM cells was higher than that of EC9706 and Eca109 cells (Figure 7a). SPAG5 was significantly upregulated in EC9706/ADM and Eca109/ADM cells compared to that in EC9706 and Eca109 cells (Figure 7b). In addition, the proliferation, invasion and migration of EC9706/ADM and Eca109/ADM cells was higher than that of EC9706 and Eca109 cells and the apoptosis of EC9706/ADM and Eca109/ADM cells was lower than that of EC9706 and Eca109 cells using clone formation, transwell, and scratch-wound assays and flow cytometry analysis (Figure 7c-7f).

SPAG5 regulated the viability, proliferation, apoptosis, invasion, and migration of EC9706/ADM and Eca109/ADM cells 
The results of qRT-PCR and western blot assays showed that low SPAG5 expression was detected in siSPAG5 group and high SPAG5 expression was detected in SPAG5 group in EC9706/ADM cells (Figure 8a). SPAG5 knockout inhibited the viability, proliferation and promoted the apoptosis, whereas, SPAG5 overexpression promoted the viability, proliferation and suppressed the apoptosis using CCK-8 and clone formation assays and flow cytometry analysis (Figure 8b-8d). The results of qRT-PCR and western blot assays showed that low SPAG5 expression was detected in siSPAG5 group and high SPAG5 expression was detected in SPAG5 group in Eca109/ADM cells (Figure 8e). SPAG5 knockout inhibited the viability, proliferation and promoted the apoptosis, whereas, SPAG5 overexpression promoted the viability, proliferation and suppressed the apoptosis using CCK-8 and clone formation assays and flow cytometry analysis (Figure 8f-8h). Transwell and scratch-wound assays showed that SPAG5 knockout inhibited the invasion and migration and SPAG5 overexpression promoted the invasion and migration of EC9706/ADM and Eca109/ADM cells (Figure 9).

\section{SPAG5 regulated proteins expression and inhibited the activation of PI3K/AKT signaling pathway in EC9706/ADM cells and Eca109/ADM cells}

Western blot showed that SPAG5 knockout promoted cleaved caspase-3 expression and inhibited VEGF and MMP-2 expression, whereas, SPAG5 overexpression inhibited cleaved caspase-3 expression and promoted VEGF and MMP-2 expression in EC9706/ADM and Eca109/ADM cells (Figure 10a and 10b). Expectedly, SPAG5 knockout inhibited the activation of PI3K/AKT signaling pathway via inhibiting the phosphorylation levels of PI3K and AKT and SPAG5 overexpression activated PI3K/AKT signaling pathway via increasing the phosphorylation levels of PI3K and AKT in EC9706/ADM and Eca109/ADM cells (Figure 10c and 10d).

\section{Discussion}

The proliferation, apoptosis, migration and invasion of tumor cells are the necessary stages of tumor occurrence and development $[2,11,15,16,19]$. Drug resistance is one of the important reasons for the low survival rate of cancer patients [21]. Our group plans to find potential therapeutic targets for esophageal cancer through bioinformatics analysis, clinical sample analysis, in vivo animal experiments and in vitro cell experiments. A total of 10 hub genes, including AURKA, PLK1, TPX2, CDC6, FOXM1, TRIP13, RAD51AP1, ECT2, SPAG5, and CKS1B, were obtained from the PPI network of 93 overlapping DEGs in GSE92396, GSE17351, and GSE9982 datasets (Figure 1). It had been reported that AURKA [22], PLK1 [23], TPX2 [24], FOXM1 [25], TRIP13 [26], and CKS1B [27] were closely related to the development and progression of ESCC. However, the effects of CDC6, RAD51AP1, ECT2 and SPAG5 on the proliferation, apoptosis, invasion, migration and chemotherapy resistance in ESCC has rarely been reported. The survival rate of patients with ESCC in high CDC6, RAD51AP1, ECT2 or SPAG5 expression group was lower than that in low CDC6, RAD51AP1, ECT2 or SPAG5 expression group (Figure 2). Interestingly, CDC6, RAD51AP1, or ECT2 expression was significantly associated with pT category and pN category, respectively. SPAG5 expression was not associated with age, gender, location, differentiation, pT category, pN category and pathological staging (Table 1 and Table 2). However, SPAG5 was an 
independent prognostic factor for OS in ESCC based on univariate and multivariate analyses and logistic analysis (Table 3, Table 4 and Figure 2). In addition, ROC curve analysis showed that SPAG5 expression has good predication ability for OS risk in patients with ESCC (Figure 2). Furthermore, high SPAG5 expression was detected in ESCC tumor tissues or cell lines, compared to normal adjacent tissues or control cells (Figure 3). Our results showed that SPAG5 expression were upregulated in ESCC tumor tissues, compared to normal adjacent tissues. Therefore, the results suggested that SPAG5 played an important role in the development and progression of ESCC. Next, cell experiments in vitro and animal experiments in vivo were used to confirm the above conjecture. SPAG5 gene knockout significantly inhibited the viability, proliferation, migration, invasion and promoted apoptosis of ESCC cells. On the contrary, overexpression of SPAG5 gene significantly promoted the viability, proliferation, migration, invasion and inhibition of apoptosis of ESCC cells (Figure 4 and Figure 5). It is reported that cell proliferation, apoptosis, migration and invasion are closely related to the changes of the related protein expression $[11,15,16,19,24]$. The expression of Caspase-3, Ki67 and VEGF in tumor tissues is closely related to the proliferation and apoptosis. High expression of VEGF promotes tumor angiogenesis, promotes tumor cell proliferation and inhibits tumor cell apoptosis [28]. Matrix metalloproteinases are a series of important endopeptidase enzymes that degrade extracellular matrix and participate in the invasion and metastasis of many kinds of tumors. MMP-2 and MMP-9 are important members of the matrix metalloproteinases family [29]. Our results confirm that SPAG5 gene knockout promotes the expression of cleavedcaspase-3 and inhibits the expression of Ki67,VEGF, and MMP-2/-9. On the contrary, overexpression of SPAG5 inhibits the expression of cleavedcaspase-3 and promotes the expression of Ki67,VEGF, and MMP-2/-9. This suggests that SPAG5 can significantly regulate the proliferation, apoptosis, migration and invasion of ESCC cells, and is related to the expression of cleavedcaspase3,Ki67,VEGF, and MMP-2/-9.

$\mathrm{PI} 3 \mathrm{~K} / \mathrm{AKT}$ signaling pathway regulates cell proliferation, apoptosis, migration, invasion and angiogenesis. AKT is the only protein downstream of PI3K that can promote malignant transformation of cells [30]. Activated AKT regulates the expression of caspase-3, Ki67, VEGF, and MMP-2/-9. Many studies have shown that abnormal activation of PI3K/AKT signaling pathway plays an important role in the occurrence and development of esophageal cancer [31,32, 33]. Our results suggest that SPAG5 gene knockout inhibits the activation of PI3K/AKT signal pathway in ESCC. However, overexpression of SPAG5 further activates PI3K/AKT signal pathway in ESCC. Cell experiments in vitro and animal experiments in vivo showed that the overexpression of SPAG5 promoted the proliferation, migration, invasion and inhibition of apoptosis of ESCC cells. The effect of PI3K/AKT inhibitor was reversed (Figure 6). It is suggested that the overexpression of SPAG5 in tumor tissue promotes the malignant phenotype of tumor by activating PI3K/AKT signal pathway.

Drug resistance of tumor cells is the main reason for the failure of clinical chemotherapy [21]. We found that SPAG5 was highly expressed not only in ESCC tumor tissues and cell lines, but also in adriamycin resistant ESCC cells (Figure 7). The results showed that SPAG5 gene knockout significantly inhibited the viability, proliferation, migration, invasion and promoted apoptosis of EC9706/ADM and Eca109/ADM cells. On the contrary, overexpression of SPAG5 gene significantly promoted the viability, proliferation, 
migration, invasion and inhibition of apoptosis of EC9706/ADM and Eca109/ADM cells (Figure 8 and Figure 9). Similarly, we found that SPAG5 gene knockout significantly promoted the expression of cleavedcaspase-3 in EC9706/ADM and Eca109/ADM cells, and inhibited the expression of Ki67, VEGF, and MMP-2/-9. Overexpression of SPAG5 inhibited the expression of cleavedcaspase-3 in EC9706/ADM and Eca109/ADM cells, and promoted the expression of Ki67, VEGF, and MMP-2/-9. Unsurprisingly, SPAG 5 overexpression increases drug resistance in ESCC cells by activating PI3K/AKT signaling pathway (Figure 10). These results suggest that the effect of SPAG5 on the proliferation, apoptosis, migration and invasion of ESCC cells is consistent with that of SPAG5 on the proliferation, apoptosis, migration and invasion of adriamycin resistant ESCC cells.

\section{Conclusion}

SPAG5 was an independent prognostic factor for OS in the patients with ESCC. High SPAG5 expression was detected in ESCC tumor tissues and cell lines and promoted the viability, proliferation, invasion, migration, and ADM resistance and inhibited its apoptosis in ESCC cells via activating PI3K/AKT signaling pathway.

\section{Abbreviations}

\begin{tabular}{ll} 
DAVID & the Database for Annotation, Visualization and Integrated Discovery \\
\hline PPI network & protein-protein interaction network \\
\hline STRING & the Search Tool for the Retrieval Interacting Genes \\
\hline ESCC & Esophageal squamous cell carcinoma \\
\hline GEO database & Gene Expression Omnibus database \\
\hline SPAG5 & Sperm-associated antigen 5 \\
\hline DEGs & The differentially expression genes \\
\hline OS & overall survival \\
\hline ROC & receiver operating characteristic \\
\hline AUC & area under the ROC curve \\
\hline GO & Gene Ontology \\
\hline KEGG & Kyoto Encyclopedia of Genes and Genomes \\
\hline ADM & adriamycin
\end{tabular}

\section{Declarations}


All patients gave written informed consent before operation. The study was approved by the Ethics Committee of the Cancer Center of The First Affiliated Hospital of Zhengzhou University. All the procedures of animal experiments were approved by the Animal Care and Use Committee of Zhengzhou University.

\section{Consent for publication}

Not applicable.

\section{Availability of data and material}

The datasets generated/analyzed during the current study are available.

\section{Competing interests}

The authors declare that they have no competing interests.

\section{Funding}

This work was supported by grants from the Medical Science and Technology Research Project of Henan Province of China (No. 201702010) and the Key Scientific Research Projects of the Higher Education Institutions of Henan Province of China (No. 20A320065).

\section{Authors' contributions}

Yan Yan and Fangbin Zhang wrote the main manuscript and analyzed the data. Yan Yan, Fangbin Zhang and Qiaoli Yi performed the experiments. Yan Yan and Kun Zhou designed the study. All authors read and approved the final manuscript.

\section{Acknowledgements}

No

\section{References}

1. Short MW, Burgers KG, Fry VT. Esophageal Cancer. Am Fam Physician. 2017;95(1):22-8.

2. Zhang SS, Xie X, Wen J, Luo KJ, Liu QW, Yang H, et al. TRPV6 plays a new role in predicting survival of patients with esophageal squamous cell carcinoma. Diagn Pathol. 2016;11:14.

3. Holscher AH, DeMeester TR, Schmidt H, Berlth F, Bollschweiler E. Propensity score-matched comparison between open and minimal invasive hybrid esophagectomy for esophageal adenocarcinoma. Langenbecks Arch Surg. 2020.

4. Tai WP, Nie GJ, Chen MJ, Yaz TY, Guli A, Wuxur A, et al. Hot food and beverage consumption and the risk of esophageal squamous cell carcinoma: A case-control study in a northwest area in China. Medicine (Baltimore). 2017;96(50):e9325. 
5. Zhang Z, Xu L, Di X, Zhang C, Ge X, Sun X. A retrospective study of postoperative radiotherapy for locally advanced esophageal squamous cell carcinoma. Ann Palliat Med. 2019;8(5):708-16.

6. Huang W, Wu L, Liu X, Long H, Rong T, Ma G. Preoperative serum C-reactive protein levels and postoperative survival in patients with esophageal squamous cell carcinoma: a propensity score matching analysis. J Cardiothorac Surg. 2019;14(1):167.

7. Lin GS, Luo HC, Fu ZC, Liao SG, Cai LJ, Zhu JF. Does brain radiotherapy improve survival in patients with esophageal squamous cell carcinoma with brain metastasis? Ann Palliat Med. 2020.

8. Du F, Sun Z, Jia J, Yang Y, Yu J, Shi Y, et al. Development and Validation of an Individualized Nomogram for Predicting Survival in Patients with Esophageal Carcinoma after Resection. J Cancer. 2020;11(14):4023-9.

9. Takahashi K, Sato S, Ota Y, Watanabe T, Tachibana S, Suda T, et al. [A Case of Local Remnant Esophageal Cancer after Chemotherapy Getting Complete Response by Radiotherapy]. Gan To Kagaku Ryoho. 2020;47(3):510-2.

10. Booka E, Haneda R, Ishii K, Kawakami T, Tsushima T, Yasui H, et al. Appropriate Candidates for Salvage Esophagectomy of Initially Unresectable Locally Advanced T4 Esophageal Squamous Cell Carcinoma. Ann Surg Oncol. 2020.

11. Xu G, Zhang Y, Li N, Wu Y, Zhang J, Xu R, et al. LBX2-AS1 up-regulated by NFIC boosts cell proliferation, migration and invasion in gastric cancer through targeting miR-491-5p/ZNF703. Cancer Cell Int. 2020;20:136.

12. He J, Green AR, Li Y, Chan SYT, Liu DX. SPAG5: An Emerging Oncogene. Trends Cancer. 2020.

13. Abdel-Fatah TMA, Agarwal D, Liu DX, Russell R, Rueda OM, Liu K, et al. SPAG5 as a prognostic biomarker and chemotherapy sensitivity predictor in breast cancer: a retrospective, integrated genomic, transcriptomic, and protein analysis. Lancet Oncol. 2016;17(7):1004-18.

14. Chen W, Chen X, Li S, Ren B. Expression, immune infiltration and clinical significance of SPAG5 in hepatocellular carcinoma: A gene expression-based study. J Gene Med. 2020;22(4):e3155.

15. Wang L, Cao L, Wen C, Li J, Yu G, Liu C. LncRNA LINC00857 regulates lung adenocarcinoma progression, apoptosis and glycolysis by targeting miR-1179/SPAG5 axis. Hum Cell. 2020;33(1):195204.

16. Yang T, Tian S, Wang L, Wang Y, Zhao J. MicroRNA-367-3p overexpression represses the proliferation and invasion of cervical cancer cells through downregulation of SPAG5-mediated Wnt/beta-catenin signalling. Clin Exp Pharmacol Physiol. 2020;47(4):687-95.

17. Zhu C, Menyhart O, Gyorffy B, He X. The prognostic association of SPAG5 gene expression in breast cancer patients with systematic therapy. BMC Cancer. 2019;19(1):1046.

18. Ansari D, Andersson R, Bauden MP, Andersson B, Connolly JB, Welinder C, et al. Protein deep sequencing applied to biobank samples from patients with pancreatic cancer. J Cancer Res Clin Oncol. 2015;141(2):369-80.

19. Zhang H, Li S, Yang X, Qiao B, Zhang Z, Xu Y. miR-539 inhibits prostate cancer progression by directly targeting SPAG5. J Exp Clin Cancer Res. 2016;35:60. 
20. Qiu Y, Lo JCK, Kwok KCW, Mason AJ, Lam JKW. Modification of KL4 Peptide Revealed the Importance of Alpha-Helical Structure for Efficient Small Interfering RNA Delivery. Nucleic Acid Ther. 2020.

21. Xavier CPR, Caires HR, Barbosa MAG, Bergantim R, Guimaraes JE, Vasconcelos MH. The Role of Extracellular Vesicles in the Hallmarks of Cancer and Drug Resistance. Cells. 2020;9(5).

22. Belkhiri A, El-Rifai W. Advances in targeted therapies and new promising targets in esophageal cancer. Oncotarget. 2015;6(3):1348-58.

23. Hu M, Zhang Q, Tian XH, Wang JL, Niu YX, Li G. IncRNA CCAT1 is a biomarker for the proliferation and drug resistance of esophageal cancer via the miR-143/PLK1/BUBR1 axis. Mol Carcinog. 2019;58(12):2207-17.

24. Yang C, Shen S, Zheng X, Ye K, Ge H, Sun Y, et al. Long non-coding RNA LINC00337 induces autophagy and chemoresistance to cisplatin in esophageal squamous cell carcinoma cells via upregulation of TPX2 by recruiting E2F4. Faseb j. 2020;34(5):6055-69.

25. Cheng L, Wang Q, Tao X, Qin Y, Wu Q, Zheng D, et al. FOXM 1 induces Vasculogenic mimicry in esophageal cancer through beta-catenin /Tcf4 signaling. Diagn Pathol. 2020;15(1):14.

26. Di S, Li M, Ma Z, Guo K, Li X, Yan X. TRIP13 upregulation is correlated with poor prognosis and tumor progression in esophageal squamous cell carcinoma. Pathol Res Pract. 2019;215(6):152415.

27. Li Z, Zhou Y, Tu B, Bu Y, Liu A, Kong J. Long noncoding RNA MALAT1 affects the efficacy of radiotherapy for esophageal squamous cell carcinoma by regulating Cks1 expression. J Oral Pathol Med. 2017;46(8):583-90.

28. Itatani Y, Kawada K, Yamamoto T, Sakai Y. Resistance to Anti-Angiogenic Therapy in CancerAlterations to Anti-VEGF Pathway. Int J Mol Sci. 2018;19(4).

29. Karmakar D, Maity J, Mondal P, Shyam Chowdhury P, Sikdar N, Karmakar P, et al. E2F5 promotes prostate cancer cell migration and invasion through regulation of TFPI2, MMP-2 and MMP-9. Carcinogenesis. 2020.

30. Ma Z, Lou S, Jiang Z. PHLDA2 regulates EMT and autophagy in colorectal cancer via the PI3K/AKT signaling pathway. Aging (Albany NY). 2020;12.

31. Sheng J, Deng X, Zhang Q, Liu H, Wang N, Liu Z, et al. PAR-2 promotes invasion and migration of esophageal cancer cells by activating MEK/ERK and PI3K/Akt signaling pathway. Int J Clin Exp Pathol. 2019;12(3):787-97.

32. Song Y, Liu H, Cui C, Peng X, Wang C, Tian X, et al. Silencing of Peroxiredoxin 1 Inhibits the Proliferation of Esophageal Cancer Cells and Promotes Apoptosis by Inhibiting the Activity of the PI3K/AKT Pathway. Cancer Manag Res. 2019;11:10883-90.

33. Wang C, Li S, Liu J, Cheng M, Wang D, Wang Y, et al. Silencing of S-phase kinase-associated protein 2 enhances radiosensitivity of esophageal cancer cells through inhibition of PI3K/AKT signaling pathway. Genomics. 2020.

\section{Tables}


Table 1. correlation between CDC6 and RAD51AP1 expression and clinicopathological parameters of patients with ESCC

Parameters

$$
\begin{array}{ll}
\text { Number of patients } \\
\begin{array}{ll}
\text { Low CDC6 } & \text { High CDC6 } \\
\text { expression } & \begin{array}{l}
\text { expression } \\
(n=34)
\end{array} \\
(n=33)
\end{array}
\end{array}
$$

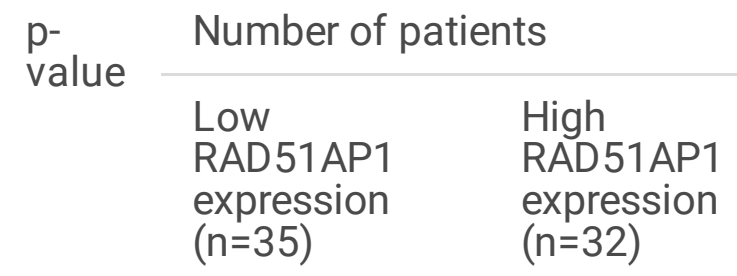

p-

\begin{tabular}{|c|c|c|c|c|c|}
\hline Age (years) & & & 0.703 & & 0.872 \\
\hline$\leq 60$ & 16 & 14 & 16 & 14 & \\
\hline$>60$ & 18 & 19 & 19 & 18 & \\
\hline Gender & & & 0.907 & & 0.890 \\
\hline Male & 18 & 17 & 18 & 17 & \\
\hline Female & 16 & 16 & 17 & 15 & \\
\hline Location & & & 0.748 & & 0.542 \\
\hline Upper & 12 & 9 & 13 & 8 & \\
\hline Middle & 11 & 13 & 11 & 13 & \\
\hline Lower & 11 & 11 & 11 & 11 & \\
\hline Differentiation & & & 0.585 & & 0.728 \\
\hline Grade1 & 10 & 10 & 10 & 10 & \\
\hline Grade2 & 11 & 14 & 12 & 13 & \\
\hline Grade3 & 13 & 9 & 13 & 9 & \\
\hline pT category & & & 0.038 & & 0.021 \\
\hline T1-2 & 22 & 13 & 23 & 12 & \\
\hline T3-4 & 12 & 20 & 12 & 20 & \\
\hline pN category & & & 0.010 & & 0.005 \\
\hline No & 23 & 12 & 24 & 11 & \\
\hline N1-3 & 11 & 21 & 11 & 21 & \\
\hline $\begin{array}{l}\text { Pathological } \\
\text { staging }\end{array}$ & & & 0.167 & & 0.239 \\
\hline$H-I I$ & 17 & 11 & 17 & 11 & \\
\hline III & 17 & 22 & 18 & 21 & \\
\hline
\end{tabular}

value

0.703

0.872 
ESCC: esophageal squamous cell carcinoma; cell division cycle 6 (CDC6); RAD51 associated protein 1 (RAD51AP1); ${ }^{\star} p<0.05$ was considered as significant.

Table 2. correlation between ETC2 and SPAG5 expression and clinicopathological parameters of patients with ESCC 
Parameters Number of patients

$$
\begin{array}{ll}
\text { Low ETC2 } & \text { High ETC2 } \\
\text { expression } & \begin{array}{l}
\text { expression } \\
(n=34)
\end{array} \\
(n=33)
\end{array}
$$

p-value Number of patients

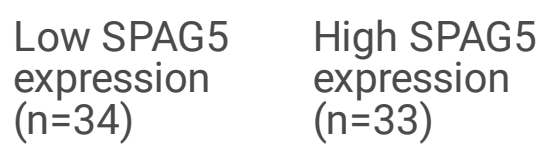

0.383

13

20

15

19

0.710 p-

value

0.912

15

18

\section{Gender}

17

17

18

0.710

Male

17

15

17

15

Female

17

15

0.898

0.532

Location

10

11

12

9

Middle

12

12

10

14

Lower

12

10

12

10

\section{Differentiation}

0.564

0.132

\begin{tabular}{ll} 
Grade1 & 12 \\
Grade2 & 11 \\
\hline Grade3 & 11 \\
\hline
\end{tabular}

8

pT category

$\begin{array}{lll}14 & 11 & 14 \\ 11 & 15 & 7\end{array}$

$8 \quad 12$

$$
0.010 * *
$$

0.273

$\begin{array}{lllll}\text { T1-2 } & 23 & 12 & 20 & 15 \\ \text { T3-4 } & 11 & 21 & 14 & 18\end{array}$

\section{pN category}

$0.038 * *$

0.113

\begin{tabular}{llccc} 
N0 & 22 & 13 & 21 & 14 \\
\hline N1-3 & 12 & 20 & 13 & 19 \\
\hline $\begin{array}{l}\text { Pathological } \\
\text { staging }\end{array}$ & & 0.918 & & \\
I-II & 14 & 14 & 15 & 13 \\
III & 20 & 19 & 19 & 20
\end{tabular}

ESCC: esophageal squamous cell carcinoma; epithelial cell transforming sequence 2 oncogene (ECT2); sperm-associated antigen 5 (SPAG5); ${ }^{*}<0.05$ was considered as significant. 
Table 3. Univariate and multivariate Cox proportional analysis with overall survival in patients with ESCC

\begin{tabular}{|c|c|c|c|c|c|c|}
\hline & Univaria & te Cox & & Multivar & te Cox & \\
\hline Parameters & $\mathrm{p}$-value & $\mathrm{HR}$ & $95 \% \mathrm{Cl}$ & $\mathrm{p}$-value & $\mathrm{HR}$ & $95 \% \mathrm{Cl}$ \\
\hline Age & 0.918 & 0.998 & $0.962 \sim 1.035$ & & & \\
\hline$(\leq 60$ vs. $>60)$ & & & & & & \\
\hline Gender & 0.691 & 0.813 & $0.293 \sim 2.253$ & & & \\
\hline (Male vs. Female) & & & & & & \\
\hline Location & & & & & & \\
\hline upper vs. Middle & 0.588 & 1.485 & $0.354 \sim 6.226$ & & & \\
\hline Middle vs. lower & 0.252 & 2.207 & $0.568 \sim 8.563$ & & & \\
\hline Differentiation & & & & & & \\
\hline Grade 1 vs. Grade2 & 0.882 & 1.098 & $0.318 \sim 3.783$ & & & \\
\hline Grade2 vs. Grade3 & 0.513 & 0.626 & $0.154 \sim 2.542$ & & & \\
\hline pT categrory & 0.048 & 3.178 & $1.008 \sim 10.021$ & & & \\
\hline (T1-2 vs. T3-4) & & & & & & \\
\hline pN category & 0.001 & 11.938 & $2.648 \sim 53.824$ & 0.002 & 14.496 & $2.677 \sim 78.493$ \\
\hline (N0 vs. N1-3) & & & & & & \\
\hline Pathological staging & 0.542 & 1.398 & $0.475 \sim 4.108$ & & & \\
\hline (I-II vs. III) & & & & 0.1 & 2.849 & $0.818 \sim 9.919$ \\
\hline CDC6 expression & 0.275 & 1.173 & $0.880 \sim 1.565$ & & & \\
\hline (low vs. high) & & & & & & \\
\hline RAD51AP1 expression & 0.289 & 1.281 & $0.902 \sim 1.409$ & & & \\
\hline (low vs. high) & & & & 0.07 & 0.722 & $0.510 \sim 1.023$ \\
\hline ECT2 expression & 0.015 & 1.806 & $1.123 \sim 2.902$ & & & \\
\hline (low vs. high) & & & & 0.09 & 1.888 & $0.899 \sim 3.965$ \\
\hline SPAG5 expression & 0.019 & 1.961 & $1.113 \sim 3.455$ & & & \\
\hline (low vs. high) & & & & 0.04 & 2.134 & $1.036 \sim 4.395$ \\
\hline
\end{tabular}


$\mathrm{HR}$, hazard ratio; $\mathrm{Cl}$, confidence interval; ESCC: esophageal squamous cell carcinoma; cell division cycle 6 (CDC6); RAD51 associated protein 1 (RAD51AP1); epithelial cell transforming sequence 2 oncogene (ECT2); sperm-associated antigen 5 (SPAG5); $\mathrm{p}^{2}<0.05$; ** $\mathrm{p}<0.01$.

Table 4. Univariate logistic regression analysis for the risk of overall survival in patients with ESCC

\begin{tabular}{llll} 
& $\beta$ & $\mathrm{p}$-value & OR $(95 \% \mathrm{Cl})$ \\
\hline Location (middle) & -1.3689 & 0.2285 & $2.54(0.021-2.16)$ \\
\hline Location (lower) & -2.3219 & 0.0751 & $9.81(0.0053-1.03)$ \\
\hline pT category (T3-4) & 3.1424 & 0.0251 & $2.32(2.23-7.18)$ \\
\hline pN category (N1-3) & 3.6764 & 0.0099 & $3.95(3.90-13.59)$ \\
\hline Pathological staging (III) & 2.0048 & 0.1051 & $7.23(0.95-19.19)$ \\
\hline ECT2 (high expression) & 0.6276 & 0.5652 & $1.87(0.24-20.33)$ \\
\hline SPAG5 (high expression) & 3.94722 & 0.0139 & $5.18(3.86-28.50)$
\end{tabular}

$\mathrm{Cl}$, confidence interval; ESCC: esophageal squamous cell carcinoma; epithelial cell transforming sequence 2 oncogene (ECT2); sperm-associated antigen 5 (SPAG5); ${ }^{*}<<0.05 ;{ }^{*} \mathrm{p}<0.01$.

\section{Figures}



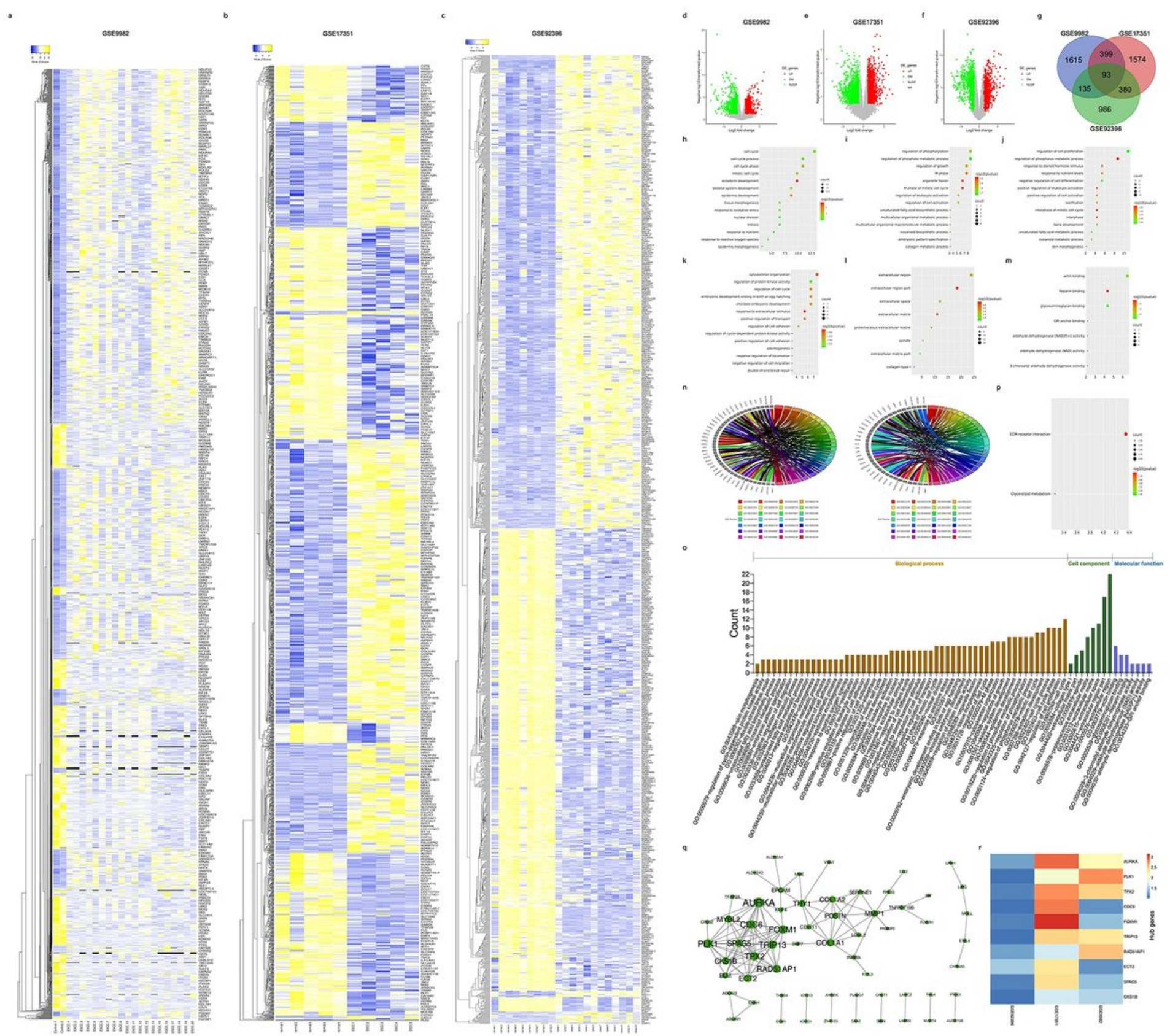

\section{Figure 1}

Identification and analysis of DEGs in ESCC (a) Heat map showing hierarchical clustering of 2242 DEGs from GSE9982 dataset between normal cell group and ESCC cell group; (b) Heat map showing hierarchical clustering of 2453 DEGs from GSE17351 dataset between normal adjacent group and ESCC tumor group; (c) Heat map showing hierarchical clustering of 1600 DEGs from GSE92396 dataset between normal adjacent group and ESCC tumor group; (d) The volcano plot of the genes in GSE9982 dataset; (e) The volcano plot of the genes in GSE17351 dataset; (f) The volcano plot of the genes in GSE92396 dataset; (g) The Venn plot of 93 overlapping genes from the DEGs of GSE9982, GSE17351 and GSE92396 datasets; (h-k) GO term analysis showed 93 overlapping genes were mainly enriched in biological process (BP) term, (I) cellular component (CC) term, and (m) molecular function (MF) term using DAVID database; (n) GO Chord plot of the relationship between the list of selected genes and their 
corresponding GO terms; (o) Count colored bar plot of GO terms. GO ID was assigned to x-axis and gene count was assigned to $y$-axis. The color intensity represented the value of terms among $B P, C C$ and $M F$ respectively; (p) KEGG pathway enrichment analysis of 93 overlapping genes using DAVID database; (q) The PPI network of 93 overlapping genes using STRING database; $(r)$ Heat map showing expression level of the top 10 hub genes from the PPI network in GSE9982, GSE17351, and GSE92396 datasets.

$$
\text { a }
$$
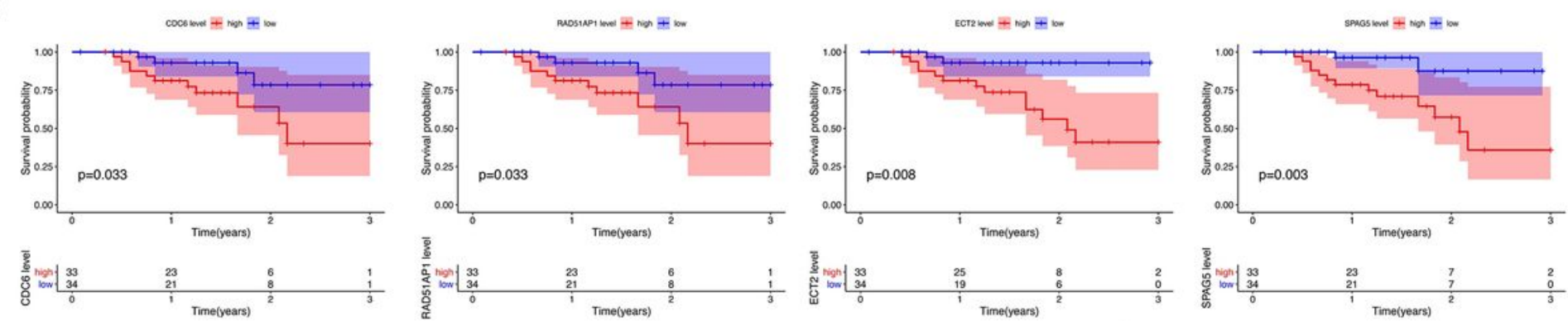

b
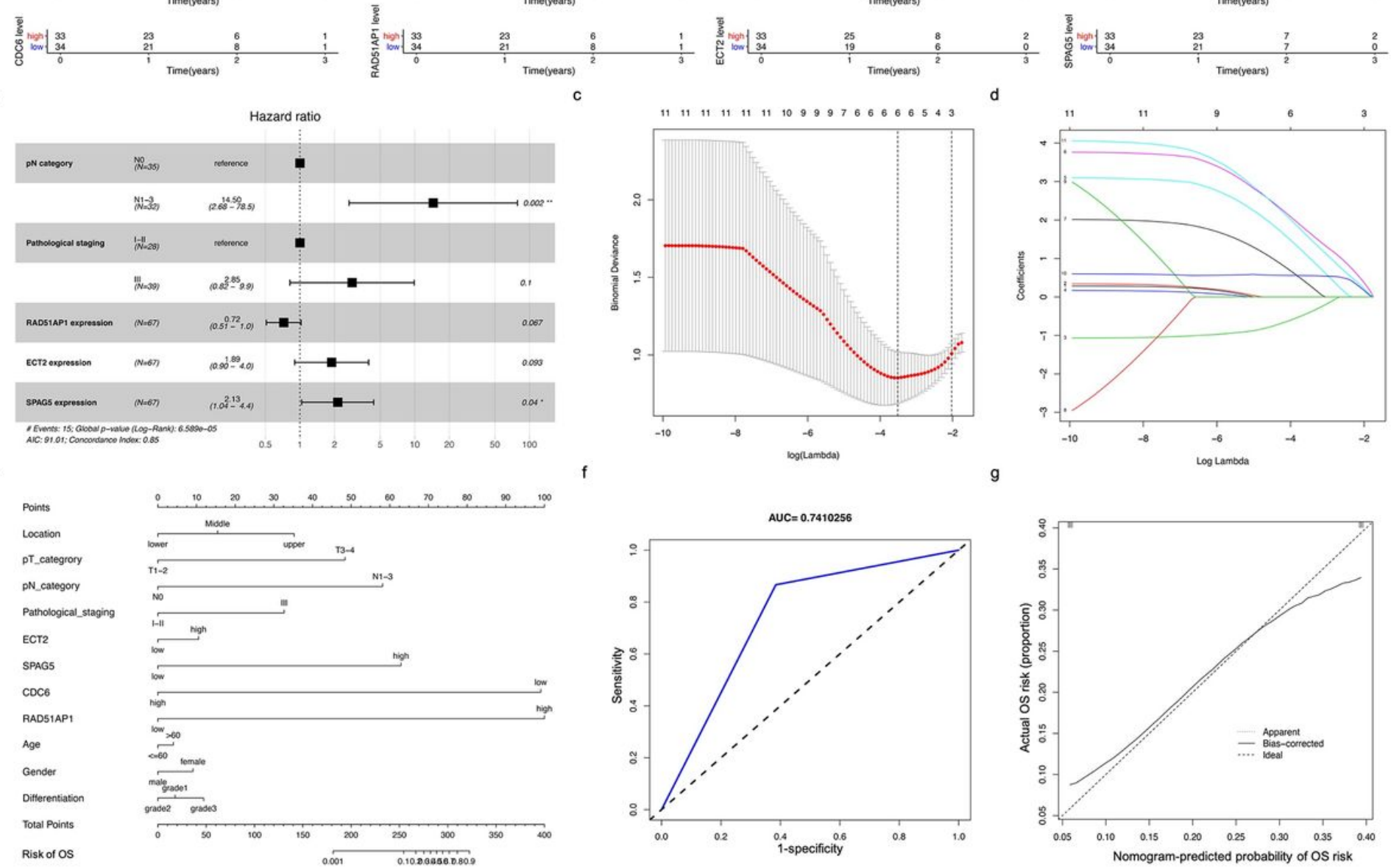

\section{Figure 2}

Clinical analysis of CDC6, RAD51AP1, ETC2 and SPAG5 (a) The relationship between CDC6 expression level, RAD51AP1 expression level, ETC2 expression level, or SPAG5 expression level and survival rate of patient with ESCC was analyzed using Kaplan-Meier analysis. (b) forest plots showing the multivariate analysis of COX regression for overall survival; (c) Optimal parameter (lambda) selection in the LASSO model used fivefold cross-validation via minimum criteria. The partial likelihood deviance (binomial deviance) curve was plotted versus log(lambda). Dotted vertical lines were drawn at the optimal values by using the minimum criteria and the $1 \mathrm{SE}$ of the minimum criteria (the 1-SE criteria); (d) LASSO coefficient profiles of the 11 features. A coefficient profile plot was produced against the log(lambda) sequence. Vertical line was drawn at the value selected using fivefold cross-validation, where optimal 
lambda resulted in five features with nonzero coefficients; (e) Developed risk of OS nomogram; (f) The ROC curve of SPAG5 to predict the risk of OS in patients with ESCC; $(\mathrm{g})$ Calibration curves of the risk of OS nomogram prediction in the cohort.

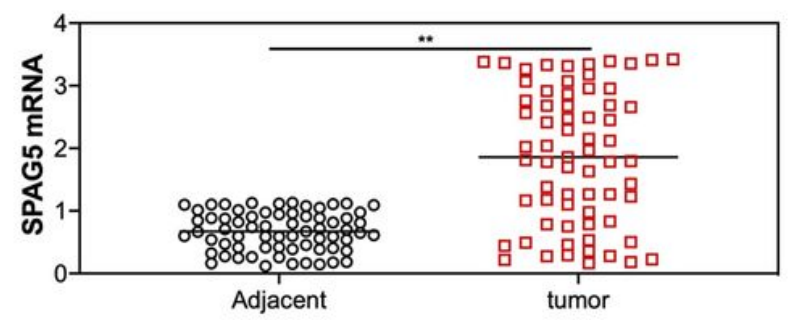

b
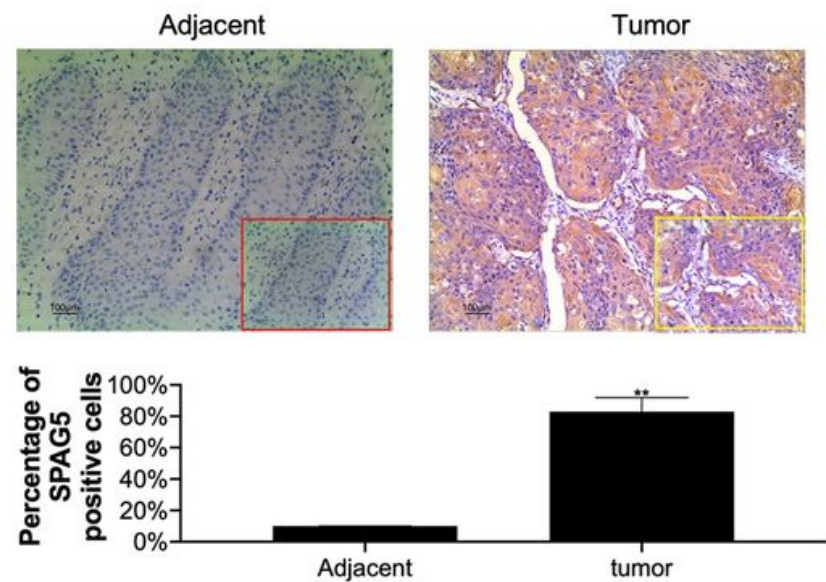

d

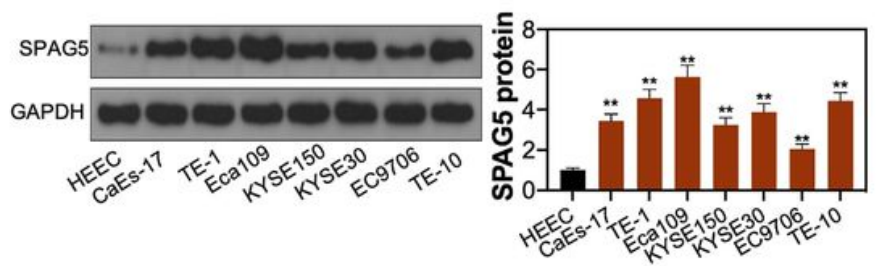

C
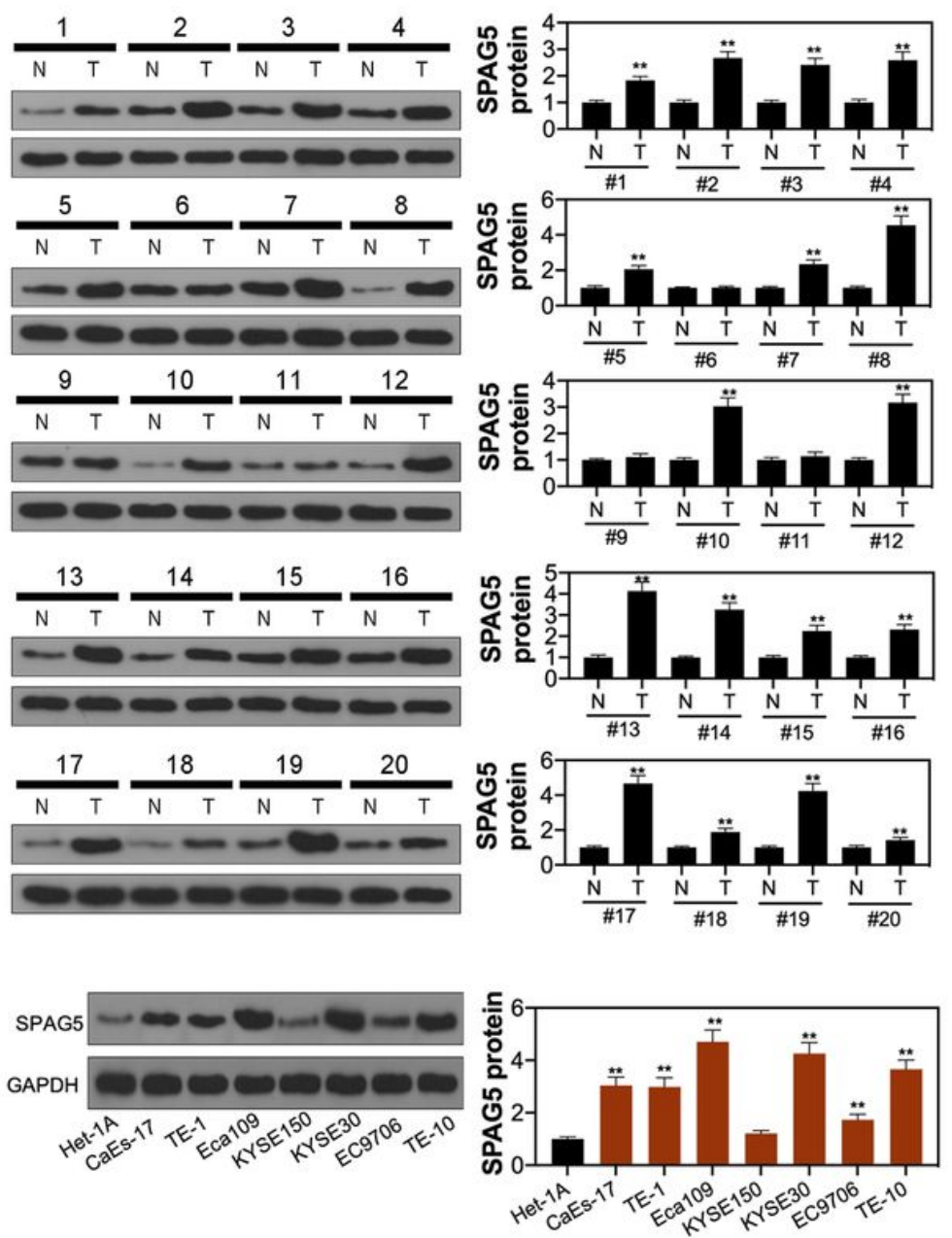

Figure 3

High SPAG5 expression was detected in ESCC tumor tissues and cell lines (a) the mRNA expression of SPAG5 in tumor tissues of patients with ESCC was detected by qRT-PCR; the protein expression of SPAG5 in tumor tissues of patients with ESCC was detected by (b) IHC assay and (c) western blot; (d and e) the protein expression of SPAG5 in ESCC cell lines was detected by western blot. GAPDH was used as a load control. Data are presented as the mean \pm standard deviation. ${ }^{* *} \mathrm{p}<0.01 \mathrm{vs}$. adjacent/HEEC/Het-1A group. 

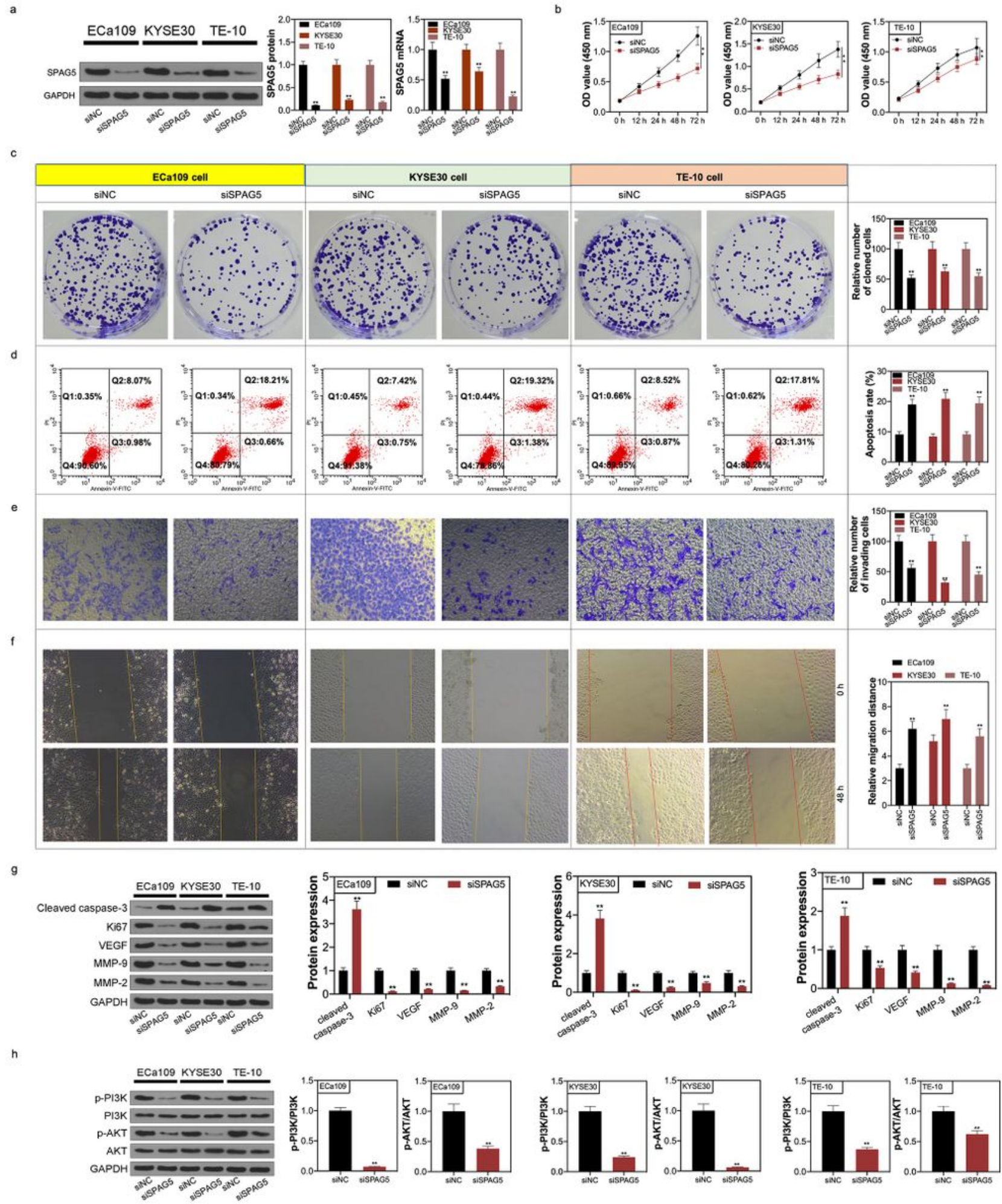

Figure 4

SPAG5 knockout inhibited the viability, proliferation, invasion and migration, and promoted the apoptosis, regulated proteins expression and suppressed the activation of PI3K/AKT signaling pathway in Eca109, KYSE30, and TE-10 cells After siRNA targeting SPAG5 was transfected into Eca109, KYSE30, and TE-10 cells, (A) SPAG5 expression in protein and mRNA levels, the cell viability, proliferation, apoptosis, invasion, and migration were detected by (a) western blot, qRT-PCR assay, (b) CCK-8 assay, (c) clone formation 
assay, (d) flow cytometry analysis, (e) transwell assay, and (f) scratch-wound assay; the protein expression cleaved caspase-3, Ki67, VEGF, and MMP-2/-9 (g) and the phosphorylation levels of PI3K and AKT (h) were detected by western blot. GAPDH was used as a load control. Data are presented as the mean \pm standard deviation. ${ }^{*} \mathrm{p}<0.01$ vs. siNC group.

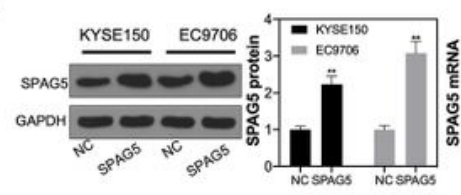

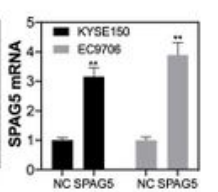
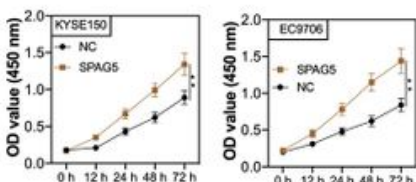
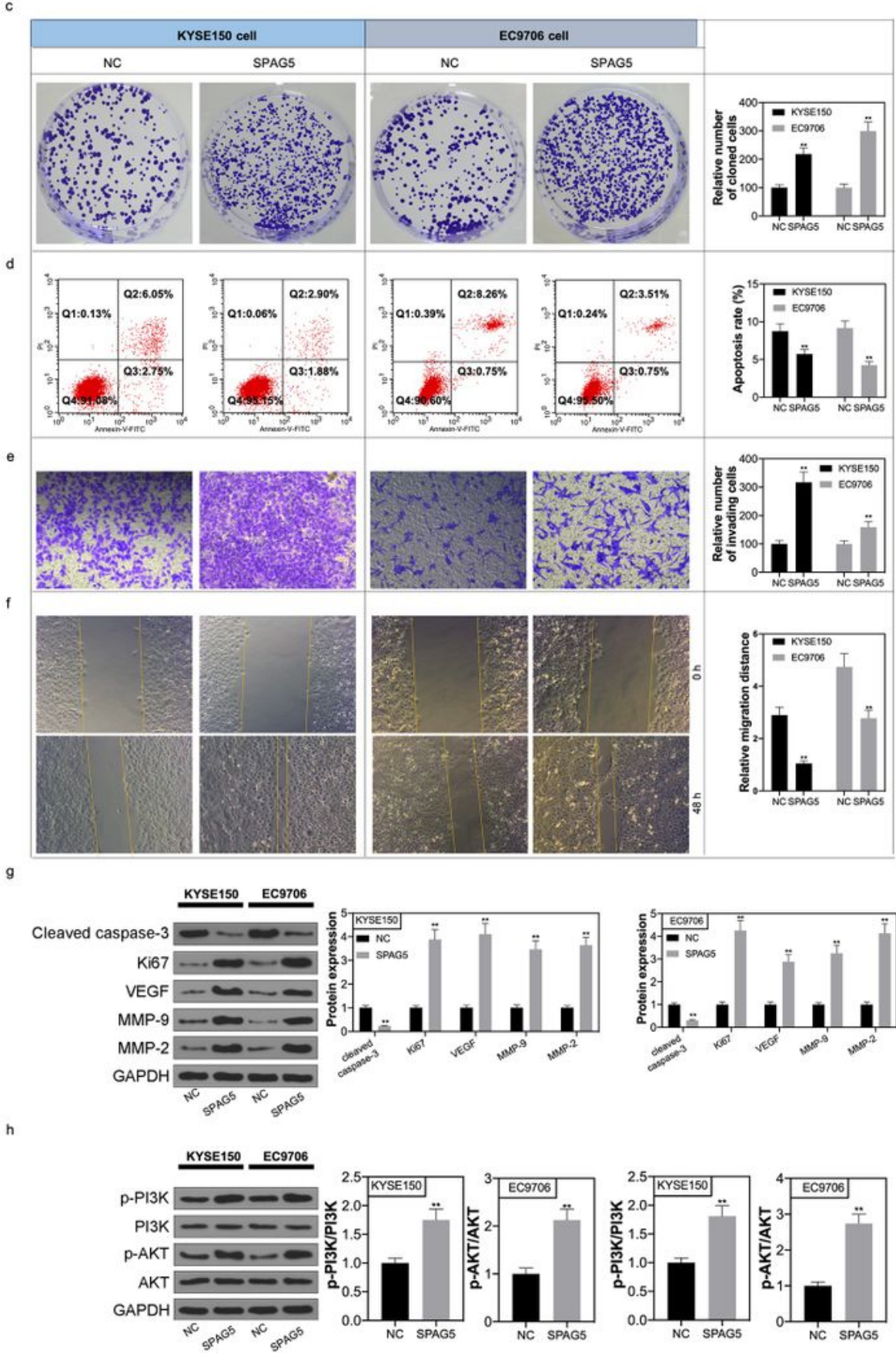

Figure 5 
SPAG5 overexpression promoted the viability, proliferation, invasion and migration, and suppressed the apoptosis, regulated proteins expression and activated PI3K/AKT signaling pathway in KYSE150 and EC9706 cells After SPAG5 expressing plasmids were transfected into KYSE150 and EC9706 cells, (A) SPAG5 expression in protein and mRNA levels, the cell viability, proliferation, apoptosis, invasion, and migration were detected by (a) western blot, qRT-PCR assay, (b) CCK-8 assay, (c) clone formation assay, (d) flow cytometry analysis, (e) transwell assay, and (f) scratch-wound assay; the protein expression cleaved caspase-3, Ki67, VEGF, and MMP-2/-9 (g) and the phosphorylation levels of PI3K and AKT (h) were detected by western blot. GAPDH was used as a load control. Data are presented as the mean \pm standard deviation. ${ }^{*} \mathrm{p}<0.01$ vs. NC group.

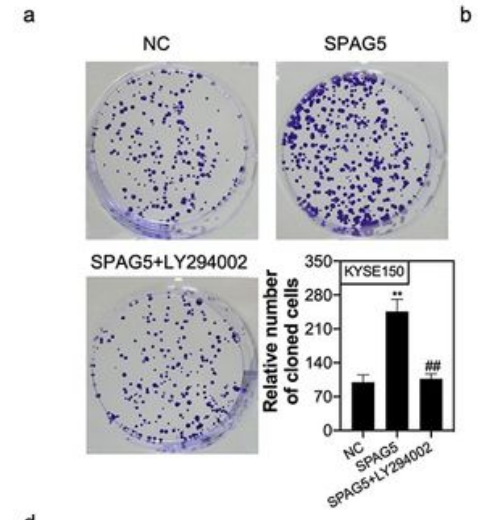

d

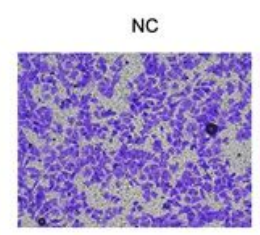

NC

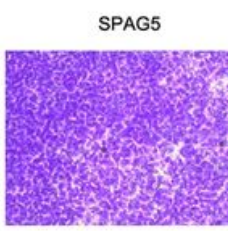

SPAG5
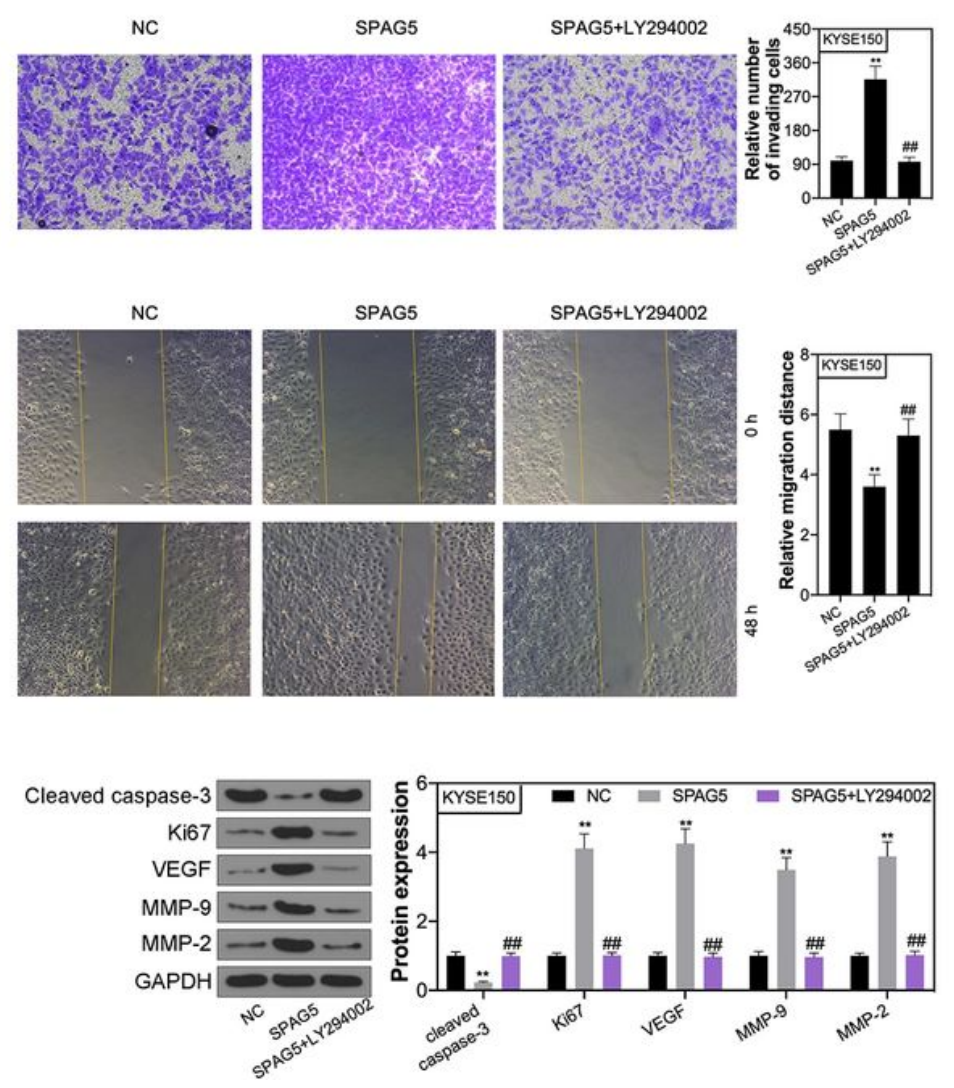
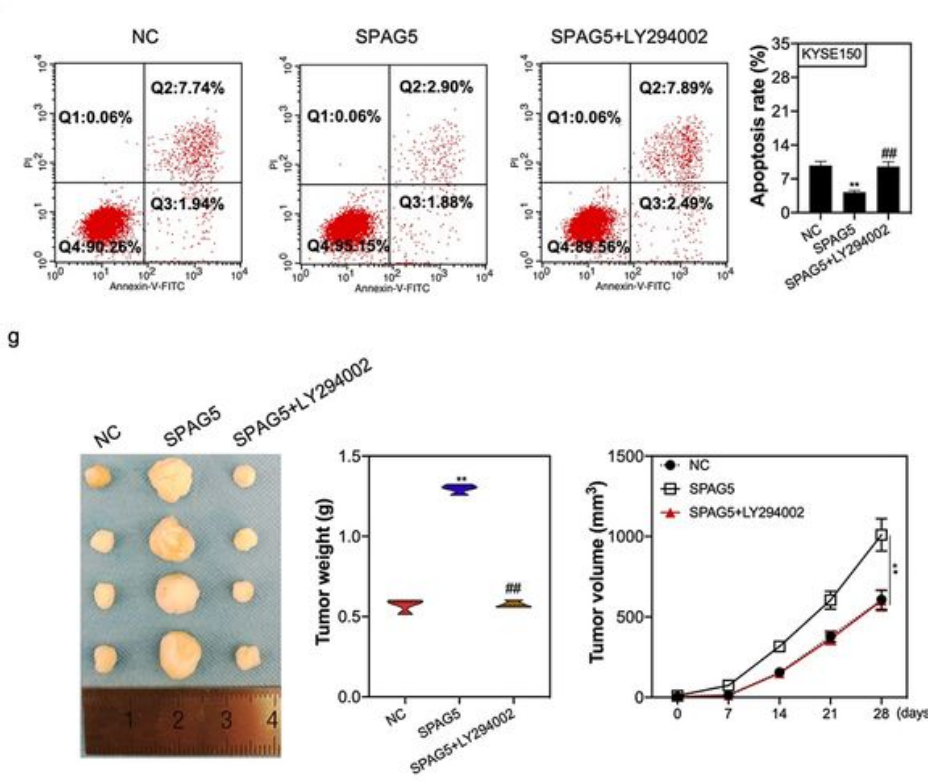

h
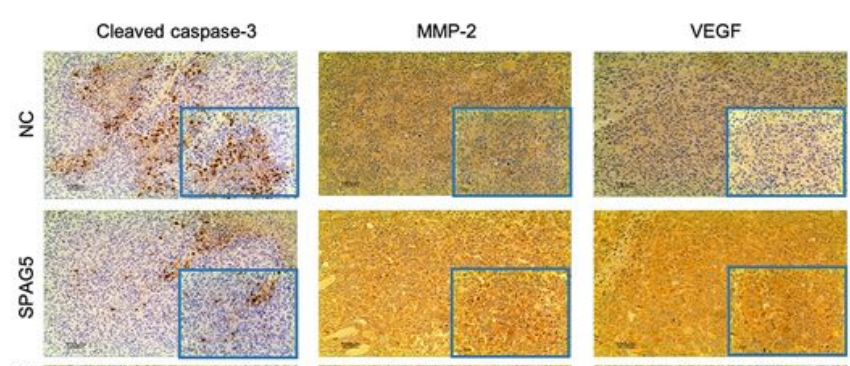

ণั
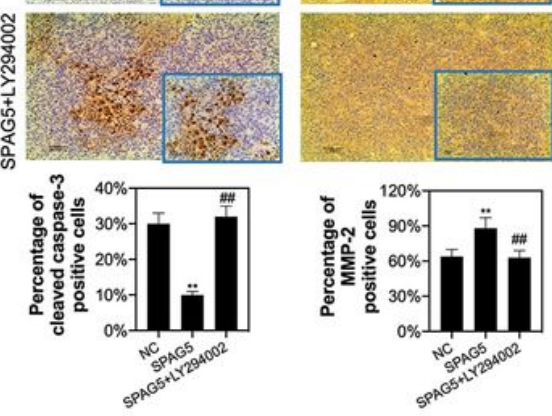

\section{Figure 6}

High SPAG5 expression promoted the growth and metastasis of ESCC in vivo and in vitro via activating PI3K/AKT signaling pathway After SPAG5 expressing plasmids were transfected into LY294002 pre- 
treated KYSE150 cells, the cell proliferation, viability, apoptosis, invasion, migration, and the protein expression of cleaved caspase-3, Ki67, VEGF, and MMP-2/-9 were detected by (a) clone formation assay, (b) CCK-8 assay, (c) flow cytometry analysis, (d) transwell assay, (e) scratch-wound assay, and (f) western blot; KYSE150 cells transfected with SPAG5 expressing plasmids or NC were subcutaneously injected into the nude mice, then LY294002 $(1.5 \mathrm{mg} / \mathrm{kg} /$ day $)$ subcutaneously injected to each immunodeficient mice, (g) all nude mice were sacrificed and the tumors were collected after 28 day, the volume and weight of the tumors were determined, $(\mathrm{h})$ the expression of cleaved caspase-3, MMP-2, and VEGF in the tumors collected from different groups were determined using IHC staining. GAPDH was used as a load control. Data are presented as the mean \pm standard deviation. ${ }^{* \star} p<0.01$ vs. NC group and $\# \# p<0.01$ vs. SPAG 5 group. 
a
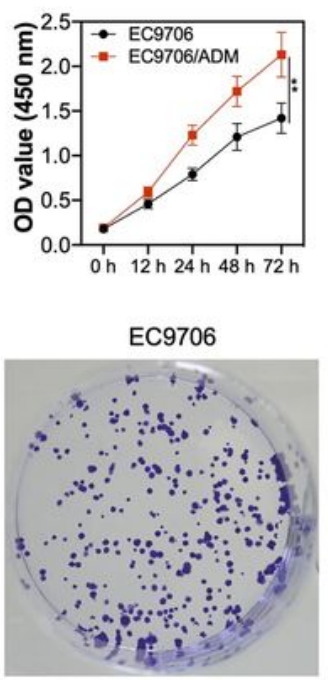

d
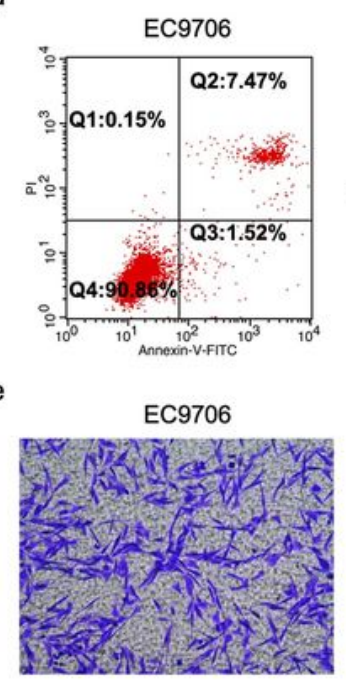

f
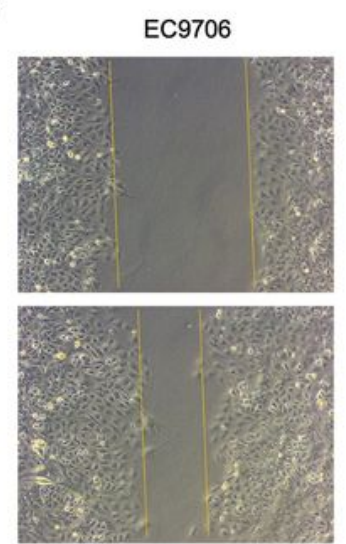

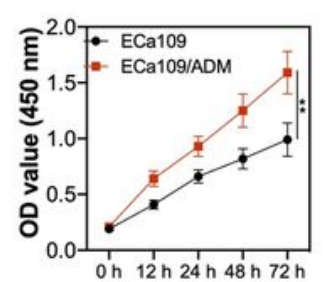

EC9706/ADM

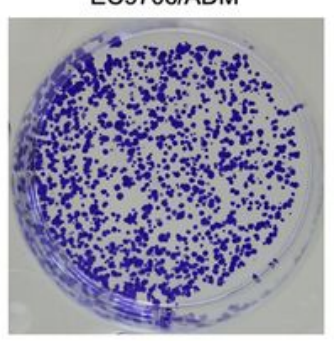

EC9706/ADM

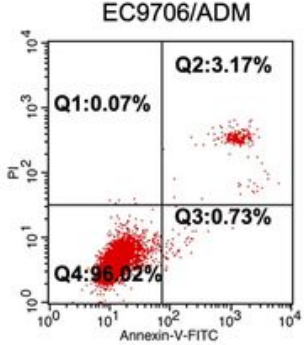

EC9706/ADM

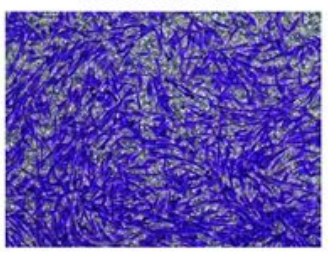

EC9706/ADM
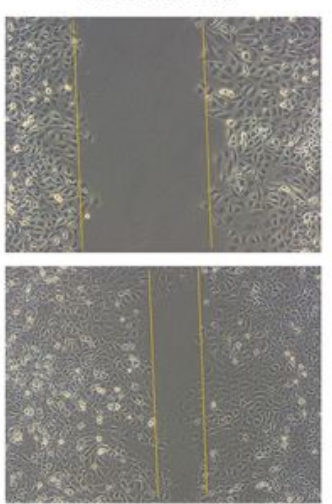

b
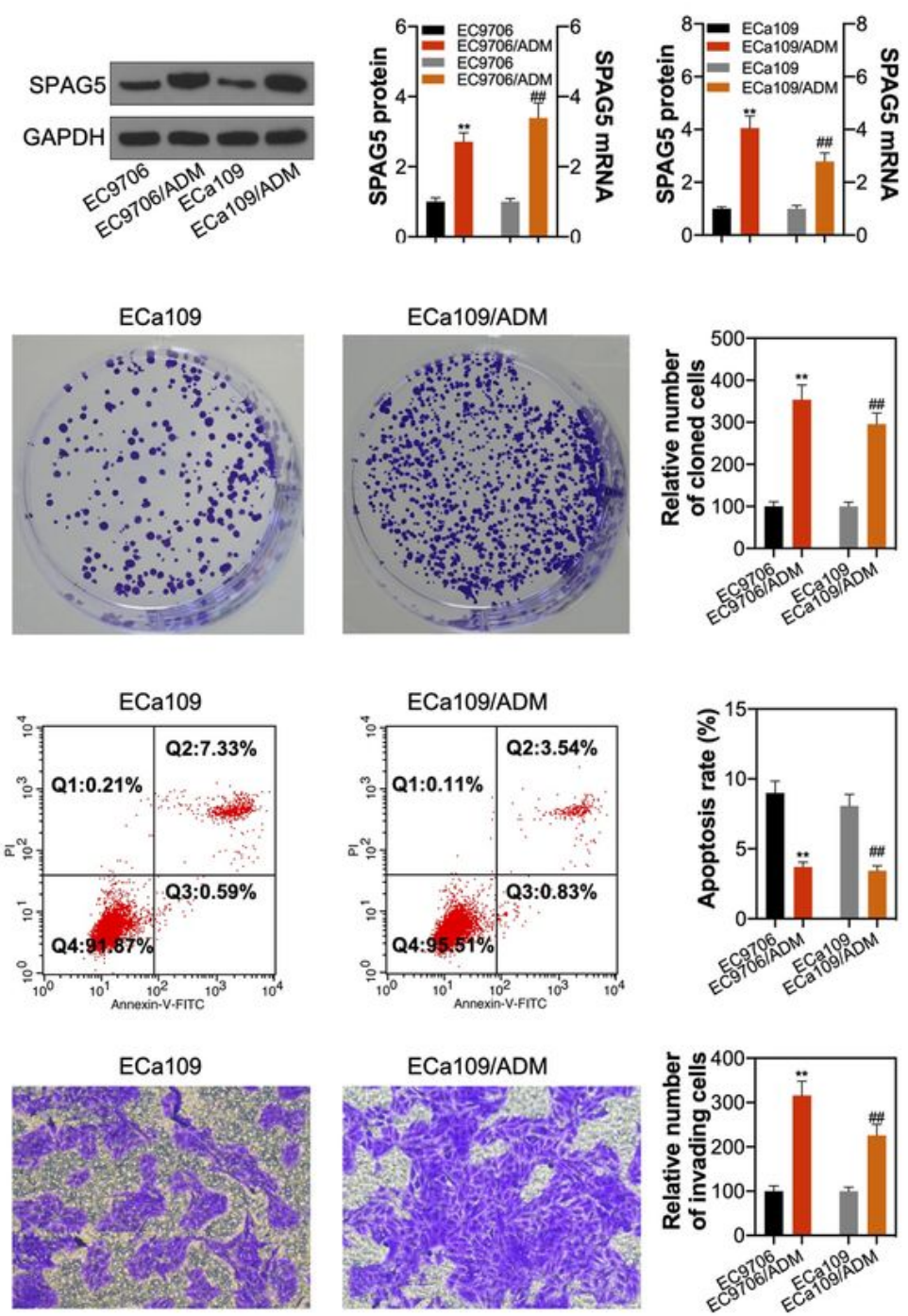

ECa109
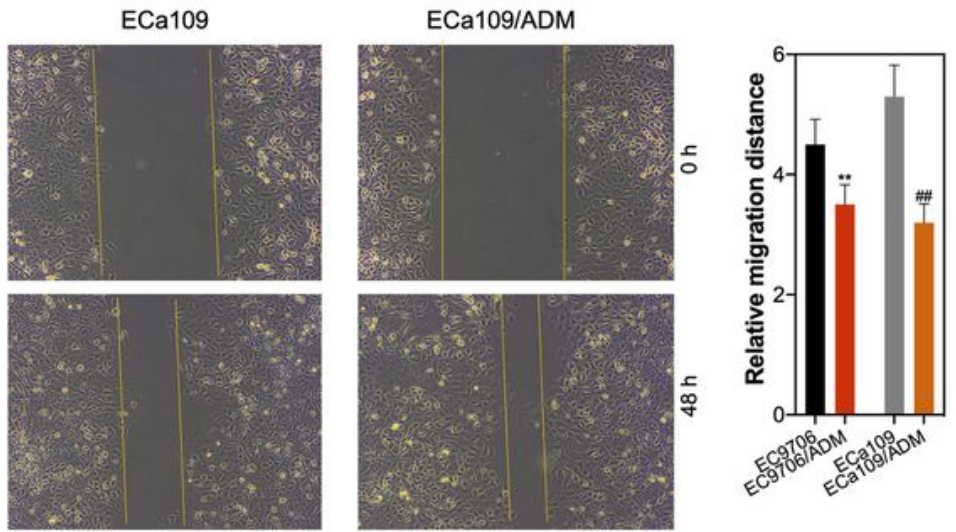

\section{Figure 7}

High SPAG5 expression was detected in ADM resistance of ESCC cells (a) CCK-8 assay was used to detect the viability between EC9706 and EC9706/ADM cells or between Eca109 and Eca109/ADM cells at 12, 24, 48 and $72 \mathrm{~h}$; SPAG5 expression, the proliferation, apoptosis, invasion, and migration was detected by (b) western blot, (c) clone formation assay, (d) flow cytometry analysis, (e) transwell assay, and (f) scratch-wound assay between EC9706 and EC9706/ADM cells or between Eca109 and Eca109/ADM 
cells. GAPDH was used as a load control. Data are presented as the mean \pm standard deviation. ${ }^{\star *} \mathrm{p}<0.01$ vs. EC9706/ Eca109 group.

c
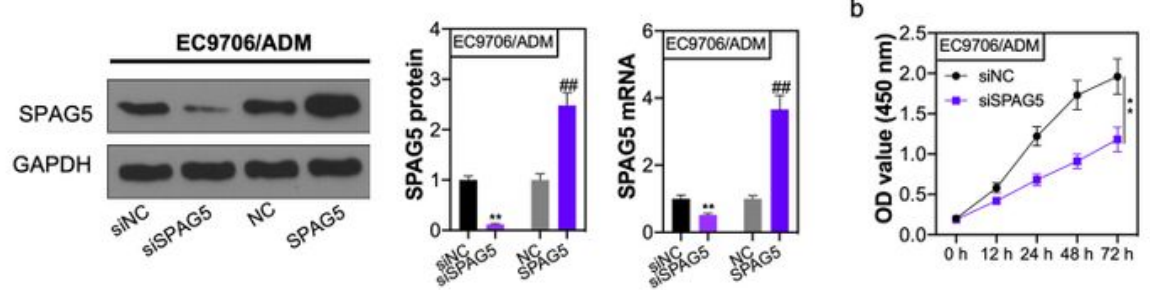

NC
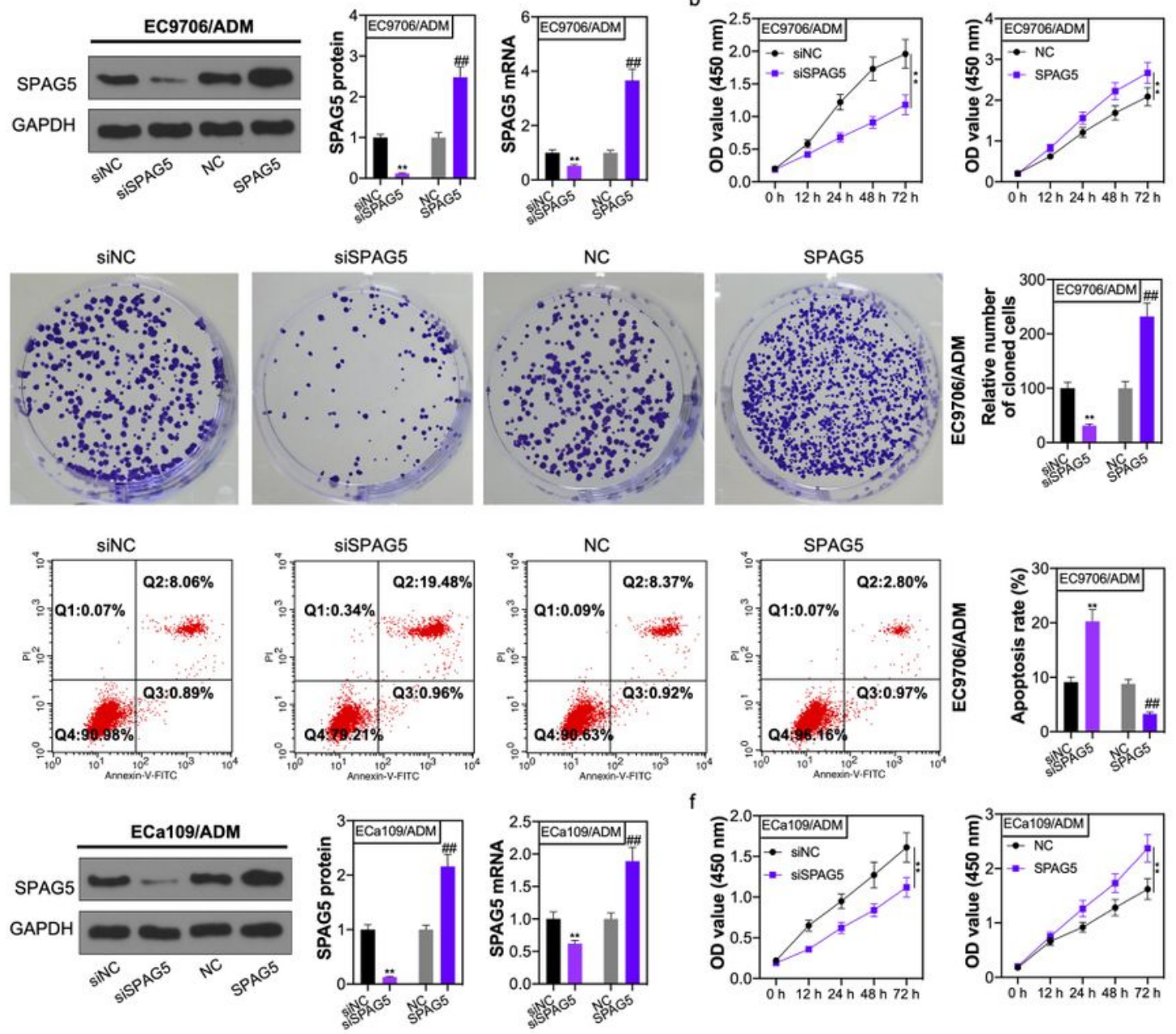

g
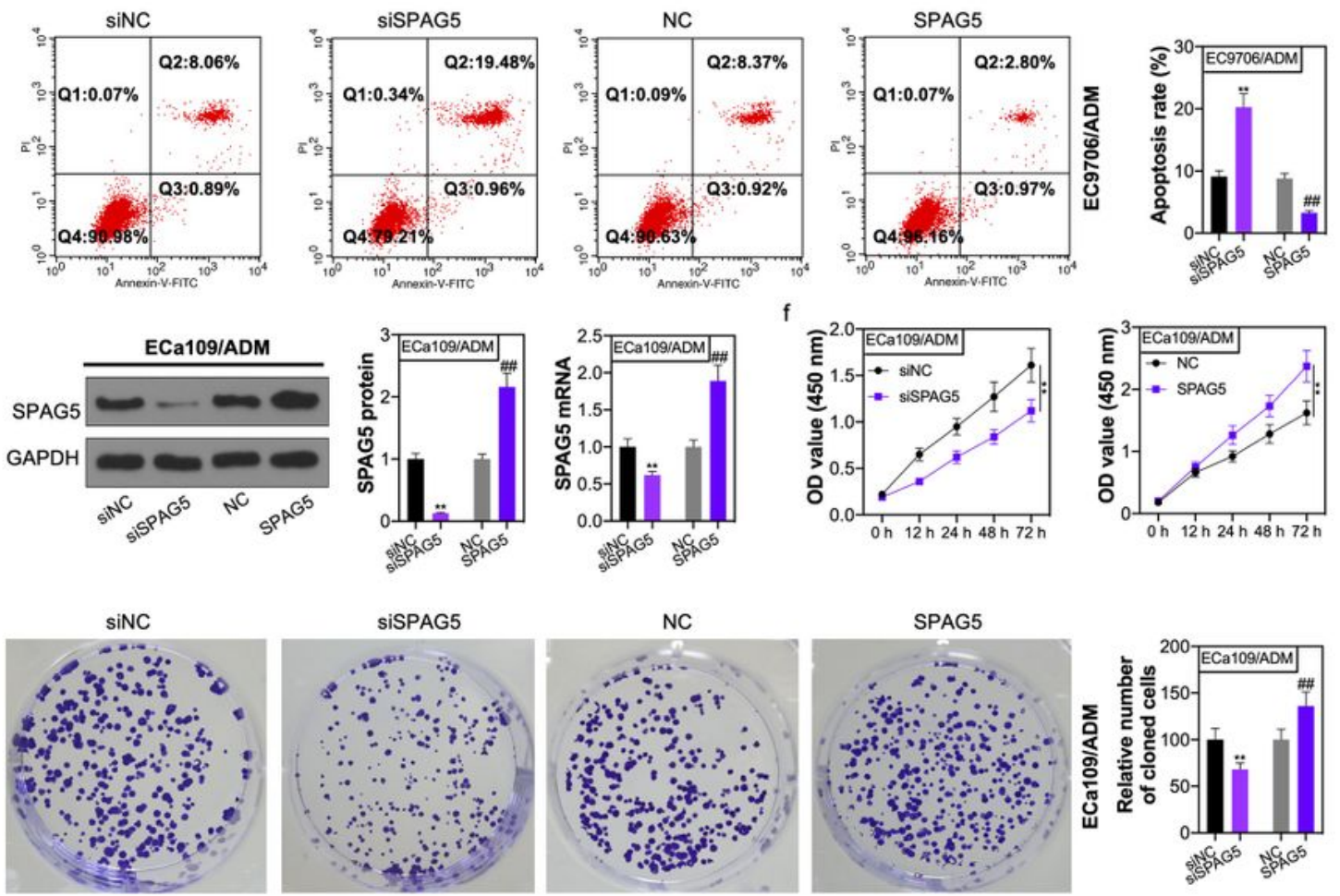

SPAG5
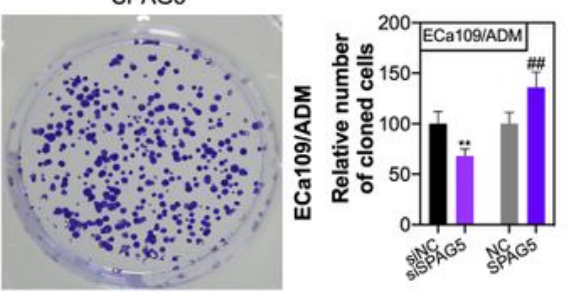

h
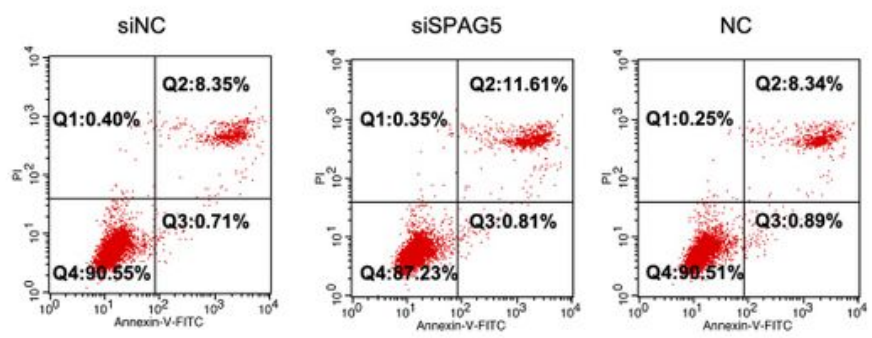

SPAG5

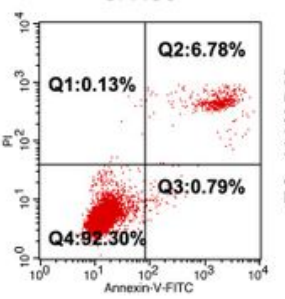

Figure 8

SPAG5 regulated the viability, proliferation, and apoptosis of EC9706/ADM and Eca109/ADM cells After siRNA targeting SPAG5 or SPAG5 expressing plasmids was transfected into EC9706/ADM cells, SPAG5 expression in protein and mRNA levels, the cell viability, proliferation, and apoptosis were detected by (a) 
western blot, qRT-PCR assay, (b) CCK-8 assay, (c) clone formation assay, and (d) flow cytometry analysis; After siRNA targeting SPAG5 or SPAG5 expressing plasmids was transfected into Eca109/ADM cells, SPAG 5 expression in protein and mRNA levels, the cell viability, proliferation, and apoptosis were detected by (e) western blot, qRT-PCR assay, (f) CCK-8 assay, (g) clone formation assay, and (h) flow cytometry analysis. GAPDH was used as a load control. Data are presented as the mean \pm standard deviation. ${ }^{* *} p$ $<0.01$ vs. siNC/NC group.

a
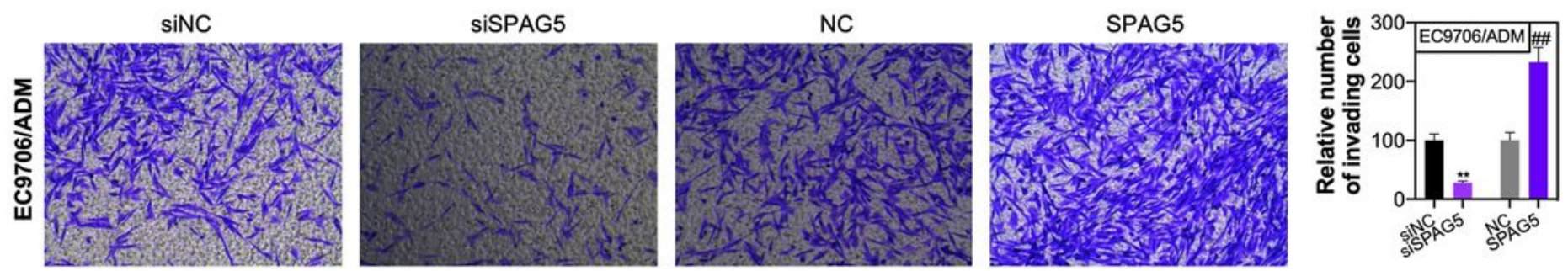

b
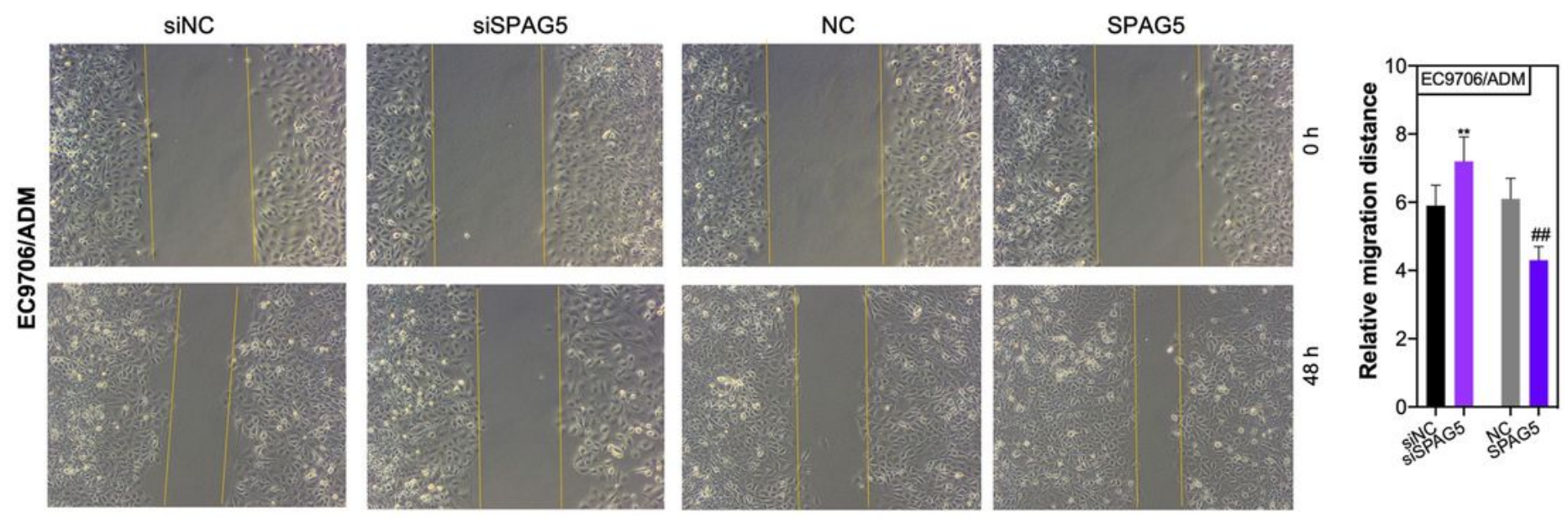

C
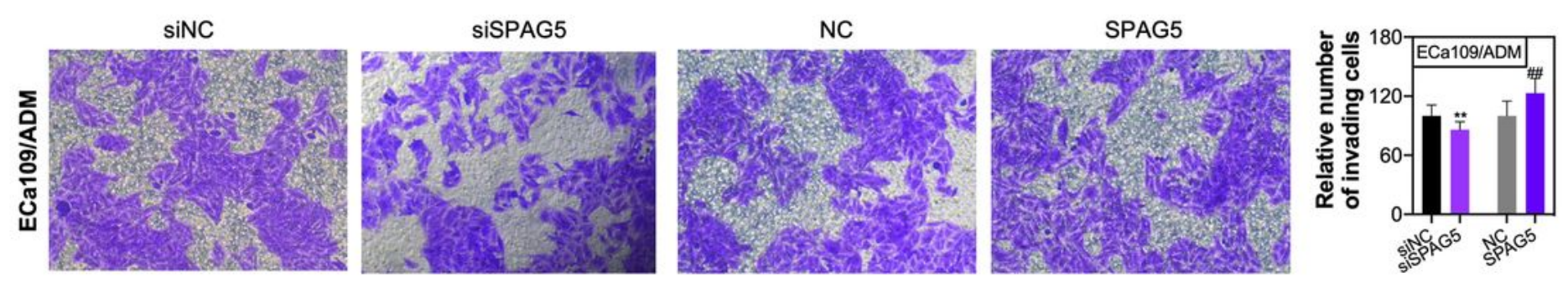

d
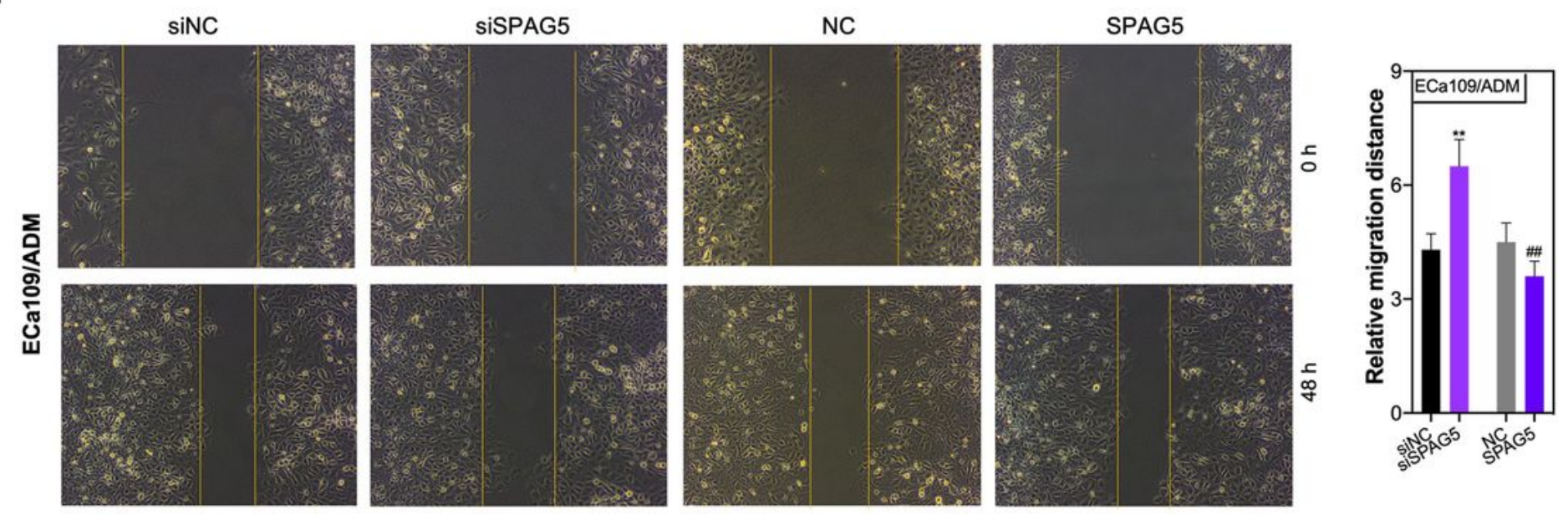

Figure 9 
SPAG5 regulated the invasion and migration of EC9706/ADM and Eca109/ADM cells After siRNA targeting SPAG5 or SPAG5 expressing plasmids was transfected into EC9706/ADM cells, the invasion and migration were detected by (a) transwell and (b) scratch-wound assays; After siRNA targeting SPAG5 or SPAG5 expressing plasmids was transfected into Eca109/ADM cells, the invasion and migration were detected by (c) transwell and (d) scratch-wound assays. GAPDH was used as a load control. Data are presented as the mean \pm standard deviation. ${ }^{* *} \mathrm{p}<0.01 \mathrm{vs}$. siNC/NC group.

a

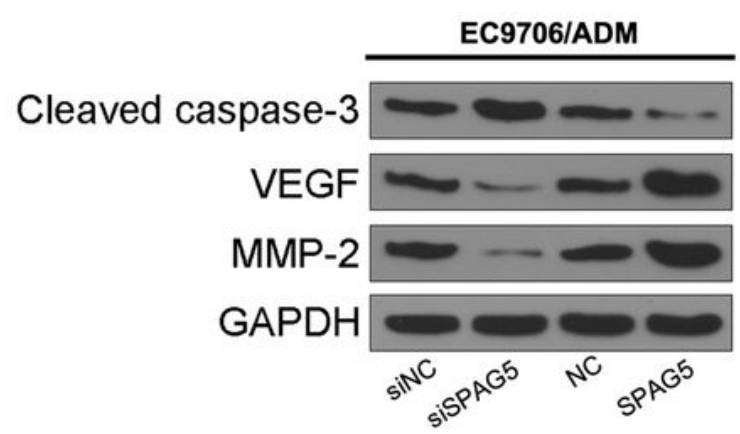

b

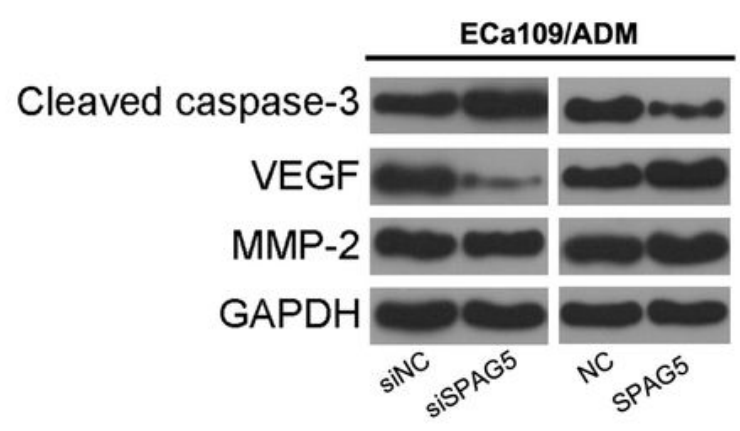

C

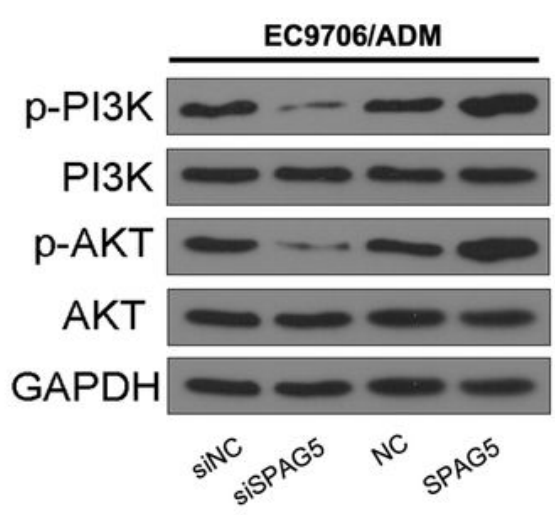

d

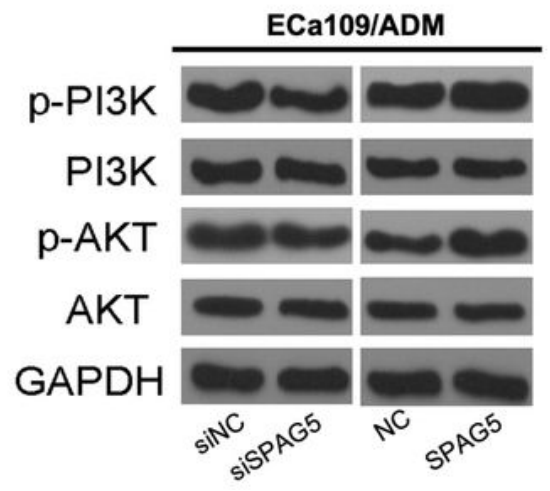

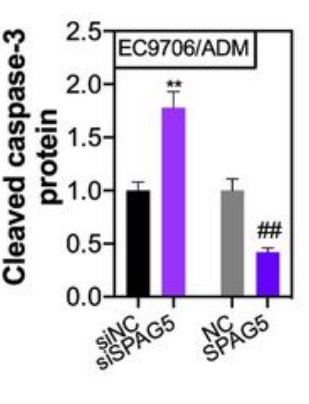
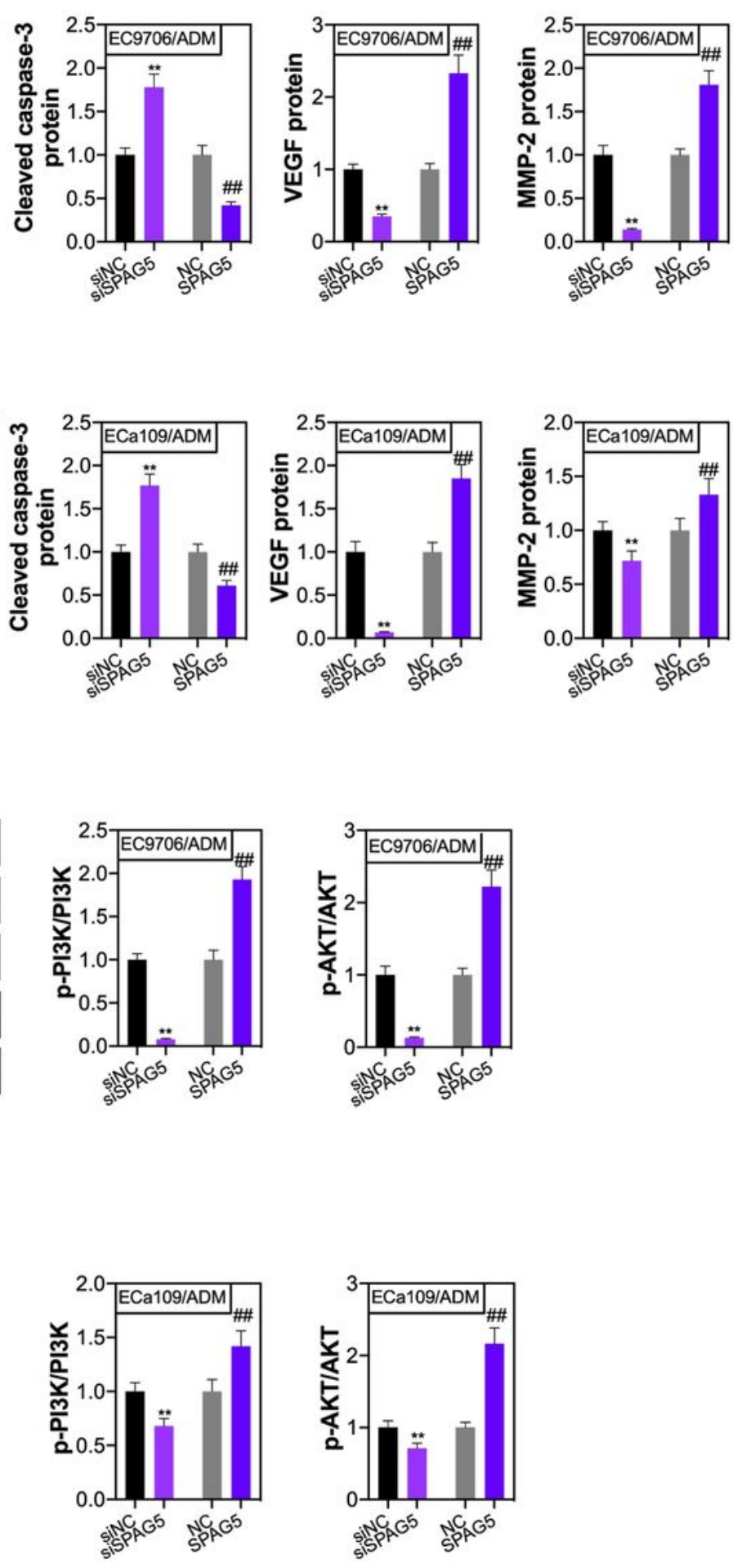

Figure 10 
SPAG5 regulated proteins expression and inhibited the activation of PI3K/AKT signaling pathway in EC9706/ADM cells and Eca109/ADM cells After siRNA targeting SPAG5 or SPAG5 expressing plasmids was transfected into EC9706/ADM and Eca109/ADM cells, the protein expression of cleaved caspase-3, VEGF, and MMP-2 by ( $a$ and $b$ ) western blot and the phosphorylation levels of PI3K and AKT by ( $c$ and d) western blot. GAPDH was used as a load control. Data are presented as the mean \pm standard deviation. $\star \star p<0.01$ vs. siNC/NC group. 